\title{
Die Änderung von Konzentration und Beweglichkeit der Ladungsträger in GaAs bei Bestrahlung mit Protonen *
}

\author{
K. Wohlleben und W. BecK \\ Forschungslaboratorium der Siemens-Schuckertwerke AG., Erlangen \\ (Z. Naturforschg. 21 a, 1057-1071 [1966] ; eingegangen am 3. März 1966) \\ Professor Dr. W. Gentner zum 60. Geburtstag gewidmet
}

\begin{abstract}
Durch Messung der Leitfähigkeit und des HalL-Effekts wurde die Abnahme der Konzentration und der Beweglichkeit der Ladungsträger in $\mathrm{n}$ - und $\mathrm{p}$-GaAs bei Bestrahlung mit 3 MeV-Protonen untersucht. Gemessen wurde die Abhängigkeit von der Bestrahlungsdosis und von der Ausgangsdotierung $\left(n_{0}=5 \cdot 10^{15}-3 \cdot 10^{18} \mathrm{~cm}^{-3}, p_{0}=2 \cdot 10^{17}-8 \cdot 10^{18} \mathrm{~cm}^{-3}\right)$. Die Träger-Vernichtungsrate erweist sich dabei als stark abhängig von der Lage des Fermi-Niveaus und von der Vorbestrahlung. Die kleinsten noch meßbaren Trägerkonzentrationen lagen bei $n \approx 10^{9} \mathrm{~cm}^{-3}$ und $p \approx 10^{10} \mathrm{~cm}^{-3}$, entsprechend einer Leitfähigkeit von $10^{-7} \Omega^{-1} \mathrm{~cm}^{-1}$. Diese Meßgrenze wird offenbar durch leitende Oberflächenschichten bestimmt. Die Extrapolation der Meßergebnisse läßt aber erwarten, daß bei fortgesetzter Bestrahlung die Trägerkonzentration von p-GaAs (Cd dotiert) sich einem Grenzwert $p^{*} \approx 1 \cdot 10^{9} \mathrm{~cm}^{-3}$ nähert und daß n-GaAs zu p-GaAs konvertiert und denselben Grenzwert erreicht $\left(E_{\mathrm{F}} * E_{\mathrm{V}}+0,58 \mathrm{eV}\right)$. Die Elektronenbeweglichkeit nimmt bei Bestrahlung monoton ab bis ca. $10^{-2}$ des Ausgangswertes. Die Löcherbeweglichkeit durchläuft dagegen ein Minimum. Wie die Analyse der Ergebnisse zeigt, müssen durch Bestrahlung in GaAs sowohl Donatoren als auch Akzeptoren erzeugt werden, und zwar in einer innerhalb etwa $0,1 \mathrm{eV}$ quasikontinuierlichen Verteilung, wobei diese Verteilung jeweils von der nächstgelegenen Bandkante bis zu einem Abstand von mindestens $0,50 \mathrm{eV}$ von dieser reicht.
\end{abstract}

In zunehmendem $\mathrm{Ma} \beta$ werden heute HalbleiterBauelemente in Fällen verwendet, bei denen sie einer Bestrahlung durch die verschiedensten Strahlungsarten ausgesetzt sind (Raumfahrt, Teilchenbeschleuniger, Reaktoren). Dadurch erhält die Frage nach dem Einfluß solcher Bestrahlungen auf die elektrischen und mechanischen Eigenschaften der Halbleiter eine große praktische Bedeutung. Die im letzten Jahrzehnt durchgeführten einschlägigen Untersuchungen galten vornehmlich den beiden Elementhalbleitern Silizium und Germanium. Durch die steigende technische Bedeutung der Verbindungshalbleiter, insbesondere $\operatorname{der} \mathrm{A}^{\mathrm{III}} \mathrm{B}^{\mathrm{V}}$-Halbleiter, gewinnen auch die in diesen Halbleitern durch Bestrahlung erzeugten Veränderungen ein wachsendes Interesse. Dies gilt besonders für $\mathrm{GaAs}$ im Hinblick auf eine mögliche Verwendung dieses Materials für Solarzellen und Transistoren.

Bestrahlungen von $\mathrm{GaAs}$ mit $1 \mathrm{MeV}$-Elektronen und mit schnellen Neutronen wurden verschiedentlich durchgeführt ${ }^{1-5}$. Es ergab sich, daß durch diese Bestrahlungen in $\mathrm{n}$ - und in $\mathrm{p}-\mathrm{GaAs}$ die Leitfähigkeit verkleinert wird. Nach starker Neutronen-

\footnotetext{
* Die in diesem Bericht mitgeteilten Forschungsarbeiten wurden mit Mitteln des Bundesministeriums für wissenschaftliche Forschung gefördert (RFT 2011).

1 R. K. Willardson, J. Appl. Phys. 30, 1158 [1959].

2 L. W. Aunerman, J. Appl. Phys. 30, 1239 [1959].
}

bestrahlung erreichte die Leitfähigkeit Werte von ${ }^{4}$ $10^{-8} \Omega^{-1} \mathrm{~cm}^{-1}$. Eine Konversion des einen Leitungstyps in den anderen wurde aber nicht beobachtet.

In der vorliegenden Arbeit wird die Änderung der Konzentration und der Beweglichkeit der Ladungsträger in $\mathrm{n}$ - und $\mathrm{p}-\mathrm{GaAs}$ bei Bestrahlung mit $3 \mathrm{MeV}$-Protonen in Abhängigkeit von der Bestrahlungsdosis und von der Ausgangsdotierung untersucht. Ferner wird der mögliche Einfluß verschiedener Dotierungselemente und Kontaktmaterialien auf das Leitfähigkeitsverhalten geprüft. Die Beeinflussung der Konzentration und der Beweglichkeit der Ladungsträger durch die Protonenbestrahlung beruht darauf, daß durch die Streuung der Protonen an den Kernen der Gitteratome diese unter geeigneten Bedingungen von ihren regulären Gitterplätzen verlagert werden. Die so erzeugten Gitterdefekte können im allgemeinen Störterme mit Donator- bzw. Akzeptorcharakter bilden, wodurch die Trägerkonzentration geändert wird. Die Gitterdefekte können zugleich auch als (geladene) Streuzentren wirken und so die Trägerbeweglichkeit beeinflussen.

3 L. W. Aukerman u. R. D. Graft, Phys. Rev. 127, 1576 [1962].

4 L. W. Aukerman, P. W. Davis, R. D. Graft u. T. S. ShilliDAY, J. Appl. Phys. 34, 3590 [1963].

5 N. A. Vitovskir, T. V. Mashovets, S. M. Ryvkin u. R. Yu. Khansevarov, Soviet Phys. Solid State 5, 2575 [1964]. 


\section{Experimentelle Methoden}

\section{Präparation der Proben}

$3 \mathrm{MeV}$-Protonen besitzen in $\mathrm{GaAs}$ eine Reichweite von $60 \mu$. Der Wirkungsquerschnitt für die Streuung der Protonen an den Kernen der Gitteratome verläuft nach der Streutheorie in guter Näherung umgekehrt proportional zur Protonenenergie (vgl. Abschn. 3). Die Dichte der erzeugten Gitterdefekte steigt daher mit wachsender Eindringtiefe der Protronen ständig an und erreicht in einer nur wenige $\mu$ tiefen Schicht unmittelbar vor dem Reichweiteende der Protonen ein steiles Maximum. Damit diese stark inhomogene Defekterzeugung am Reichweiteende der Protonen bei einer Mittelwertbildung der Defektdichte über die gesamte Probe unberücksichtigt bleiben kann, betrug die Dicke der GaAs-
Proben im Mittel 45,5 $\mu$. Die Protonen treten dann mit einer mittleren Restenergie von $1,1 \mathrm{MeV}$ aus der Rückseite der Proben aus. Wie noch gezeigt wird, unterscheiden sich die Grenzwerte der Defektdichten an der Vorder- und Rückseite der Proben dann um etwa einen Faktor 2,3.

Tab. 1 gibt eine Übersicht über die Vielfalt der untersuchten Proben. Alle Proben bestehen aus einkristallinem GaAs. Als Ausgangsmaterial für die p-leitenden und die meisten n-leitenden Proben dienten jeweils 4 verschiedene tiegelfrei zonengezogene Stäbe $(6-8 \mathrm{~mm} \phi)$. Der Rest der n-leitenden Proben stammte aus 6 verschiedenen Stäben, die nach der Czochralski-Technik im Tiegel gezogen wurden (ca. $20 \mathrm{~mm} \phi)^{*}$.

Aus den tiegelfrei gezogenen Stäben wurden Scheibchen mit 6-8 $\mathrm{mm}$ Durchmesser und aus den im Tiegel gezogenen Stäben quadratische Proben mit $5 \mathrm{~mm}$ Kantenlänge geschnitten. Die Proben wurden zunächst mit

\begin{tabular}{|c|c|c|c|c|c|c|c|}
\hline \multicolumn{8}{|c|}{ n-GaAs } \\
\hline Gruppe & $\begin{array}{l}n_{0} \\
\mathrm{~cm}^{-3}\end{array}$ & $\begin{array}{c}\mu_{\mathrm{e} 0} \\
\mathrm{~cm}^{2} \mathrm{~V}^{-1} \mathrm{~s}^{-1}\end{array}$ & Dotierung & $\begin{array}{l}\text { Herstel- } \\
\text { lung }^{\mathrm{a}}\end{array}$ & Kontakte & $\begin{array}{l}\text { nicht } \\
\text { geätzt }\end{array}$ & geätzt \\
\hline 1 & $5,0 \cdot 10^{15}$ & 2630 & undotiert & $\mathrm{F}$ & $\begin{array}{l}\text { Sn } \\
\text { In }\end{array}$ & $\begin{array}{l}\times \\
\times\end{array}$ & $\times$ \\
\hline 2 & $2,5 \cdot 10^{16}$ & 4050 & undotiert & F & $\begin{array}{l}\text { Sn } \\
\text { In }\end{array}$ & $\begin{array}{l}\times \\
\times\end{array}$ & $\times$ \\
\hline 3 & $5,0 \cdot 10^{16}$ & 3880 & undotiert & F & $\begin{array}{l}\text { Sn } \\
\text { In }\end{array}$ & $\begin{array}{l}\times \\
\times\end{array}$ & $\times$ \\
\hline 4 & $1,5 \cdot 10^{17}$ & 3010 & $\begin{array}{c}\text { Te } \\
\text { undotiert }\end{array}$ & $\begin{array}{l}\mathrm{F} \\
\mathrm{C}\end{array}$ & $\begin{array}{l}\text { In } \\
\text { In }\end{array}$ & $\begin{array}{l}\times \\
\times\end{array}$ & $x$ \\
\hline 5 & $3,5 \cdot 10^{17}$ & 3320 & undotiert & $\mathrm{C}$ & In & $x$ & \\
\hline 6 & $1,0 \cdot 10^{18}$ & 2730 & $\begin{array}{c}\mathrm{Te} \\
\text { undotiert }\end{array}$ & $\begin{array}{l}\mathrm{F} \\
\mathrm{C}\end{array}$ & $\begin{array}{l}\text { In } \\
\text { In }\end{array}$ & $\begin{array}{l}\times \\
\times\end{array}$ & $x$ \\
\hline 7 & $1,5 \cdot 10^{18}$ & 2050 & $\begin{array}{c}\mathrm{Si}, \mathrm{Te} \\
\mathrm{Te}\end{array}$ & $\stackrel{\mathrm{C}}{\mathrm{F}}$ & $\begin{array}{l}\text { Sn, In } \\
\text { In }\end{array}$ & $x$ & $\times$ \\
\hline 8 & $3,0 \cdot 10^{18}$ & 2240 & $\mathrm{Te}$ & $\mathrm{F}$ & In & $x$ & $x$ \\
\hline \multicolumn{8}{|c|}{ p-GaAs } \\
\hline 1 & $2,0 \cdot 10^{17}$ & 194 & $\mathrm{Cd}$ & $\mathrm{F}$ & $\underset{\mathrm{In}}{\mathrm{Sn} / \mathrm{Zn}}$ & $\begin{array}{l}\times \\
\times\end{array}$ & $x$ \\
\hline 2 & $3,0 \cdot 10^{17}$ & 198 & $\mathrm{Cd}$ & $\mathrm{F}$ & $\begin{array}{l}\mathrm{Sn} / \mathrm{Zn} \\
\mathrm{In}\end{array}$ & $\begin{array}{l}x \\
\times\end{array}$ & $x$ \\
\hline 3 & $4,3 \cdot 10^{17}$ & 189 & $\mathrm{Cd}$ & F & $\mathrm{Sn} / \mathrm{Zn}$ & $x$ & $\times$ \\
\hline 4 & $1,7 \cdot 10^{18}$ & 138 & $\mathrm{Zn}$ & F & $\mathrm{Sn} / \mathrm{Zn}$ & $x$ & $x$ \\
\hline 5 & $3,8 \cdot 10^{18}$ & 99 & $\mathrm{Zn}$ & $\mathrm{F}$ & $\mathrm{Sn} / \mathrm{Zn}$ & $x$ & $x$ \\
\hline 6 & $8,0 \cdot 10^{18}$ & 84 & $\mathrm{Zn}$ & F & $\mathrm{Sn} / \mathrm{Zn}$ & $x$ & $x$ \\
\hline
\end{tabular}

Tab. 1. Übersicht über die untersuchten GaAs-Proben. a $\mathrm{F}=$ Tiegelfrei zonengezogene Stäbe, $\mathrm{C}=\mathrm{Tiegelgezogene}$ Stäbe (Czochralski-Technik).

* Wir danken Herrn Dr. H. Merkel, daß er diese Proben zur Verfügung stellte. 
Granat (Korngröße $30 \mu$ ) auf etwa $70 \mu$ und dann mit Granat der Korngröße $8 \mu$ auf $60 \mu$ abgeschliffen. Anschließend wurde mit $\mathrm{Al}_{2} \mathrm{O}_{3}$ (Korngröße $0,3 \mu$ ) und Polierpech auf Endmaß poliert. Von allen Proben wurde die Dicke einzeln bestimmt. Diese lag zwischen 45 und $50 \mu$, bei einer mittleren Dicke von $48 \mu$. Wie Stichproben ergaben, betrug der Keilfehler über die Proben weniger als $1-2 \mu$. Die Rauhtiefe der polierten Flächen lag ebenfalls unter $1 \mu$ **.

Anschließend wurden auf den Rand der Proben 4 Kontakte in symmetrischer Anordnung bei ca. $550{ }^{\circ} \mathrm{C}$ unter Formiergas $\left(\mathrm{N}_{2}-\mathrm{H}_{2}\right.$-Gemisch) in Form kleiner Kügelchen von etwa $0,3 \mathrm{~mm}$ Durchmesser einlegiert (Legierungszeit ca. $3 \mathrm{~min}$ ). $\mathrm{n}-\mathrm{GaAs}$ wurde mit In oder $\mathrm{Sn}, \mathrm{p}-\mathrm{GaAs}$ mit In oder mit einer Legierung von $\mathrm{Sn}+$ $10 \% \mathrm{Zn}$ kontaktiert.

Um den Einfluß leitender Oberflächenschichten zu untersuchen, wurde schließlich ein Teil der kontaktierten Proben in Königswasser geätzt, wobei die Kontakte mit Picein abgedeckt waren. Es wurden beidseitig 1,5 bis $3 \mu$ abgeätzt, so daß die Dicke der geätzten Proben im Mittel $43 \mu$ betrug.

Da die Proben zur Vorbereitung für die Bestrahlungen und die elektrischen Messungen stets freitragend gehandhabt wurden, war die Bruchgefahr wegen der geringen Dicke sehr groß. Es mußte deshalb mit einer großen Anzahl von Proben gearbeitet werden (insgesamt ca. 120 Stück). An den meisten Proben konnten mehrere - an einzelnen Proben bis zu zehn - elektrische Messungen in Abhängigkeit vom integrierten Protonenfluß durchgeführt werden. Die verschiedenen Proben, die jeweils in einer der Gruppen der Tab. 1 zusammengefaßt sind, unterscheiden sich nur wenig bezüglich der Ausgangsdotierung, da sie meist aus benachbarten Scheibchen desselben Stabes stammen. Das mit „undotiert“ bezeichnete n-GaAs war nicht absichtlich dotiert, so daß die Dotierungssubstanz vorwiegend Si aus der Quarzwand der Ziehapparatur sein dürfte.

\section{Bestrahlung}

Die Bestrahlungen wurden an einem 3-MeV-V $\mathrm{V}_{\mathrm{AN} \text { DE }}$ GraAFF-Beschleuniger durchgeführt. Aus dem Ionenstrahl des Beschleunigers wurde der $\mathrm{H}_{1}{ }^{+}$-Ionenstrahl zunächst von den Molekülionen magnetisch absepariert und dann durch zwei gekreuzte magnetische Wechselfelder (Sägezahnströme mit $5 \mathrm{~Hz}$ und $200 \mathrm{~Hz}$ ) in einer Zickzacklinie nach Art eines Fernsehrasters periodisch über die zu bestrahlende Fläche abgelenkt. Die Inkonstanz der beiden Ablenkfrequenzen und der Durchmesser des Protonenstrahls von ca. $1 \mathrm{~mm}$ bewirken, $\mathrm{da} ß$ bereits nach kurzer Zeit eine homogene Ausleuchtung der bestrahlten Fläche erreicht wird, wie durch Autoradiographien bestätigt werden konnte. Die GaAsProben wurden auf die plangeschliffenen Höcker eines Halters aus V2A-Stahl aufgesetzt, wobei die nichtkontaktierte Seite der Proben dem Protonenstrahl zugewendet war (Abb. 1). Mit einer sehr dünnen Schicht aus Vakuumfett wurden die Proben auf die Höcker

** Für diese Bearbeitung der Proben sei Herrn E. Behrens und seinen Mitarbeitern besonders gedankt. „aufgeklebt“. Damit war ein gewisser Wärmekontakt zwischen Probe und Halter hergestellt. Der Probenhalter wurde auf einem mit flüssigem Stickstoff gekühlten Kryostaten befestigt und in die Vakuumkammer $\left(2 \cdot 10^{-5}\right.$ Torr $)$ eingebaut.

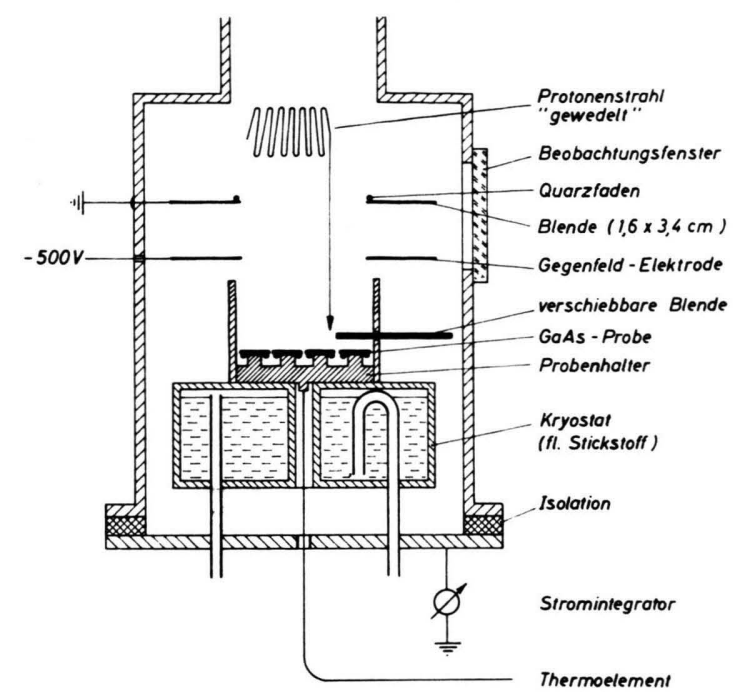

Abb. 1. Anordnung zur Bestrahlung der GaAs-Proben mit Protonen.

Eine $5 \mathrm{~cm}$ über dem Probenhalter angebrachte Rechteckblende definierte die zu bestrahlende Fläche. Der Probenhalter war als Faraday-Käfig ausgebildet. Durch eine vorgeschaltete Gegenfeld-Elektrode (Spannung $-500 \mathrm{~V}$ ) wurden die von den Protonen an Halter und Proben ausgelösten Sekundärelektronen aus der Strommessung eliminiert. Wie sich zeigte, war der Anteil an Sekundärelektronen jedoch gering. Um die Führung des Protonenstrahls auch während der Bestrahlung zu kontrollieren, wurde der Strahl allseitig so weit über den Blendenausschnitt abgelenkt, daß die den Ausschnitt einrahmenden dünnen Quarzfäden noch getroffen wurden. Das dabei vom Quarz emittierte blaue Fluoreszenzlicht konnte mit einer Fernsehkamera beobachtet werden.

Der auf den Probenhalter treffende Protonenstrom wurde mit einem Stromintegrator gemessen und zeitlich aufintegriert (Fehlanzeige 1\%). Es wurde angestrebt, stets mit konstanter Strahlstromdichte von $0,05 \mu \mathrm{A} / \mathrm{cm}^{2}$ $\mathrm{zu}$ bestrahlen. Die verschiedenen Werte des integrierten Protonenflusses wurden also durch entsprechend lange Bestrahlungszeiten erhalten. Ohne die Bestrahlungskammer zu belüften, konnten durch Vorschieben von Blenden einzelne Proben mit verschiedenen integrierten Protonenflüssen bestrahlt werden. Die thermische Belastung der Proben von $0,1 \mathrm{Watt} / \mathrm{cm}^{2}$ ließ die Temperatur des Halters zwar unverändert bei $77^{\circ} \mathrm{K}$, es wird jedoch geschätzt, daß die Temperatur der Proben während der Bestrahlung um etwa $30^{\circ}$ höher lag. Nach der Bestrahlung wurde der Kryostat auf Zimmertemperatur erwärmt und die Proben mit Petroläther vom Halter abgelöst. 


\section{Elektrische Messungen}

Die elektrische Leitfähigkeit und die HALL-Spannung wurden nach der Methode von van Der PaUw ${ }^{6}$ gemessen. Die Elektronenkonzentration $n$ und die Löcherkonzentration $p$ wurden aus den HaLL-Koeffizienten $R_{\mathrm{H}}$ bestimmt :

$$
R_{\mathrm{H}}=-\frac{r}{|e| n} \quad \text { bzw. } \quad R_{\mathrm{H}}=+\frac{r}{|e| p}
$$

( $e=$ Elementarladung). Im vorliegenden Fall lassen sich diese Beziehungen stets anwenden, da auch die kleinsten noch meßbaren Majoritätsträgerkonzentrationen um 3 Größenordnungen über der Eigenleitungskonzentration lagen. Der Faktor $r$ wurde gleich 1 gesetzt. Die HalL-Beweglichkeit $\mu$ bestimmt man in bekannter Weise nach

$$
\mu=R_{\mathrm{H}} \sigma .
$$

Bei Trägerkonzentrationen über $10^{14} \mathrm{~cm}^{-3}$ konnte mit einem Kompensator gemessen werden. Bei kleineren Konzentrationen wurde eine abgeschirmte Meßapparatur mit einem elektronischen Schwingkondensatorelektrometer mit $10^{14} \Omega$ Eingangswiderstand verwendet. Bei den stark bestrahlten Proben waren wegen der kleinen Trägerbeweglichkeiten auch die HALL-Spannungen sehr klein. Die hohen Nullspannungen mußten dann häufig mit einer fremden konstanten Gegenspannung kompensiert werden. Im allgemeinen ließen sich Elektronenkonzentrationen unter $10^{9} \mathrm{~cm}^{-3}$ und Löcherkonzentrationen unter $10^{10} \mathrm{~cm}^{-3}$ nicht mehr messen. Die Proben zeigten dann eine sehr unterschiedliche Leitfähigkeit zwischen den verschiedenen Kontakten; außerdem wurde die Messung der HaLL-Spannung durch hohe Nullspannungen verhindert, die sich nicht mehr kompensieren ließen, da sie sich laufend änderten.

Das inhomogene Leitfähigkeitsverhalten dieser hochohmigen Proben dürfte darauf zurückzuführen sein, daß die Gesamtleitfähigkeit der Proben überwiegend durch leitende Oberflächenschichten mit lokal unterschiedlicher Leitfähigkeit bestimmt wurde. Diese Annahme wird durch die Messung an einer infolge Bestrahlung sehr hochohmigen n-GaAs-Probe $\left(>10^{7} \Omega \mathrm{cm}\right)$ in verschiedenen Atmosphären gestützt. Die Leitfähigkeit war in Ozon um etwa einen Faktor 10 größer als in feuchtem Stickstoff.

Nach stärkerer Bestrahlung von n-GaAs niedriger Ausgangsdotierung (Gruppe 1 und 2) wurden öfters einzelne Kontakte so hochohmig, daß elektrische Messungen nicht mehr möglich waren. Da die Proben von der den Kontakten entgegengesetzten Seite bestrahlt wurden, lag das Reichweiteende der Protonen und damit auch der Bereich maximaler Defektdichte gerade in der Legierungszone von Kontaktmaterial und GaAs. Die hohe Defektdichte in dieser Zone dürfte dann für das Ansteigen des Kontaktwiderstandes verantwortlich sein.

${ }^{6}$ L. J. van der Pauw, Philips Res. Rept. 13, 1 [1958].

\section{Meßergebnisse}

\author{
$n-G a A s$
}

Die Abb. 2 zeigt für die verschiedenen Ausgangsdotierungen $n_{0}$ die Abnahme der Elektronenkonzentration von $\mathrm{n}-\mathrm{GaAs}$ bei Bestrahlung. Um die Elektronenkonzentration auf einen vorgegebenen Wert zu reduzieren, müssen demnach um so mehr Protonen aufgestrahlt werden, je größer $n_{0}$ ist. Nachdem die Elektronenkonzentration um etwa einen Faktor 10 gegenüber $n_{0}$ verkleinert wurde, fällt sie mit zunehmender Bestrahlung dann sehr steil ab. Der Verlauf der Kurven läßt vermuten, $d a ß n$ durch weitere Bestrahlung noch abnehmen wird. Jedoch konnten Elektronenkonzentrationen unter $10^{9} \mathrm{~cm}^{-3}$ nicht mehr gemessen werden.

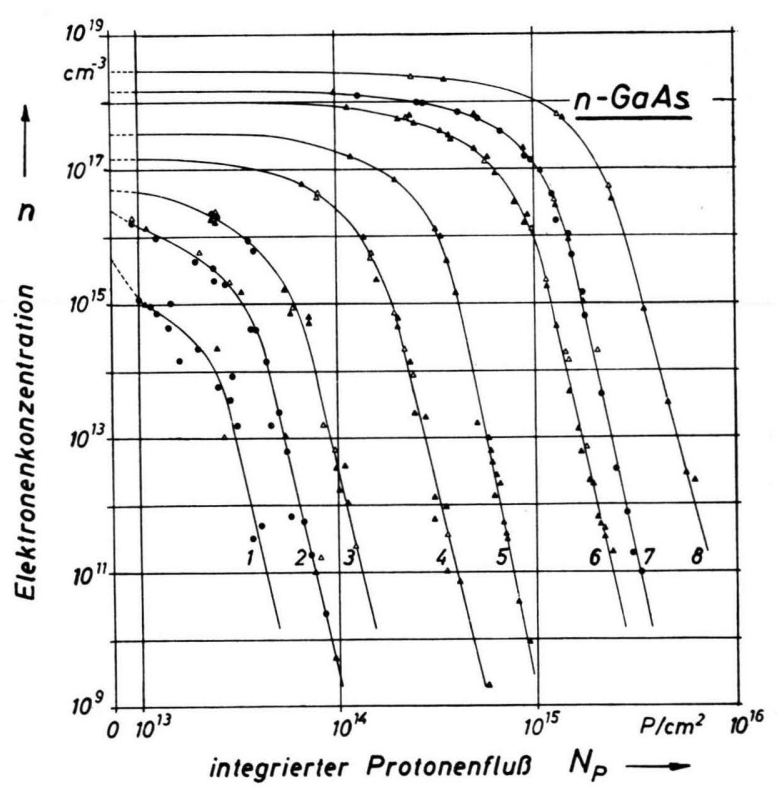

Abb. 2. Elektronenkonzentration von $\mathrm{n}-\mathrm{GaAs}$ verschiedener Ausgangsdotierung in Abhängigkeit vom integrierten Protonenfluß bei $3 \mathrm{MeV}$. ( Sn-Kontakt, nicht geätzt; $\boldsymbol{\Delta}$ In-Kontakt, nicht geätzt; $\triangle$ In-Kontakt, geätzt).

Das Verhalten der Elektronenbeweglichkeit bei Bestrahlung ist in Abb. 3 dargestellt. Die Abnahme des Verhältnisses $\mu_{\mathrm{e}} / \mu_{\mathrm{eo}}$ beginnt bei um so größerer Protonendosis und erfolgt dann um so langsamer, je größer $n_{0}$ ist. Hierin liegt eine gewisse Parallelität im Verhalten von Beweglichkeit und Konzentration der Elektronen. Jedoch besteht ein wesentlicher Unterschied darin, daß die Elektronenbeweglichkeit nur bis auf einen Wert im Bereich von $10^{-2}$ der Ausgangsbeweglichkeit abnimmt, wogegen dieselbe 


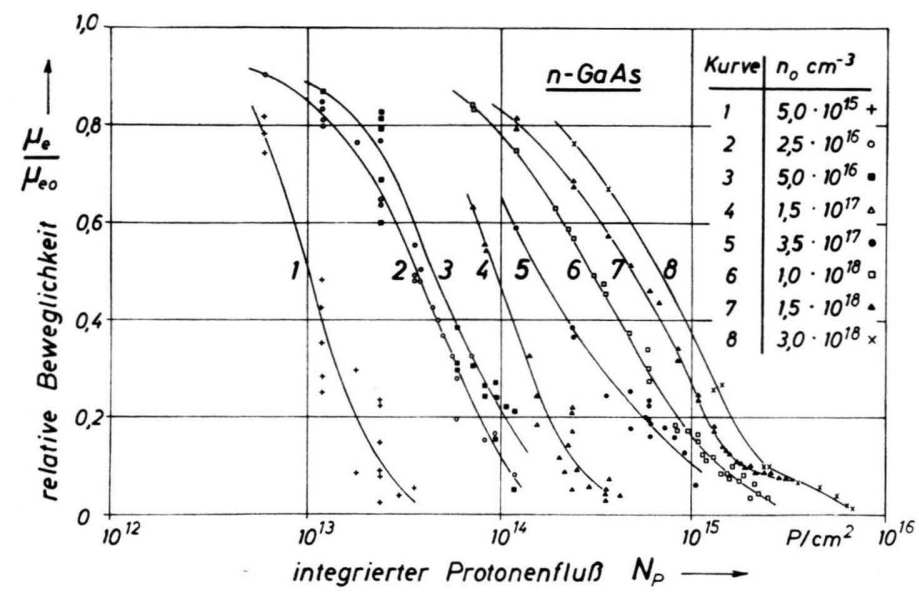

Abb. 3. Beweglichkeit der Elektronen in $\mathrm{n}-\mathrm{GaAs}$ verschiedener Ausgangsdotierung in Abhängigkeit vom integrierten Protonenfluß bei $3 \mathrm{MeV}\left(\mu_{\mathrm{eo}}=\right.$ Elektronenbeweglichkeit vor der Bestrahlung).
Protonendosis die Elektronenkonzentration um 6Größenordnungen und mehr reduziert. Sobald $\mu_{\mathrm{e}}$ auf etwa $10 \%$ von $\mu_{\mathrm{eo}}$ abgesunken ist, zeigt sich eine Tendenz zur Sättigung.

Die Abnahme der spezifischen Leitfähigkeit $\sigma$ (Abb. 4) entspricht weitgehend derjenigen von $n$, da diese Größe für die Leitfähigkeitsänderung überwiegend verantwortlich ist. Durch die Abnahme von $\mu_{\mathrm{e}}$ um maximal 2 Größenordnungen wird der Abfall von $\sigma$ nur noch beschleunigt. Die Grenze der meßbaren Leitfähigkeit liegt bei $10^{-7} \Omega^{-1} \mathrm{~cm}^{-1}$

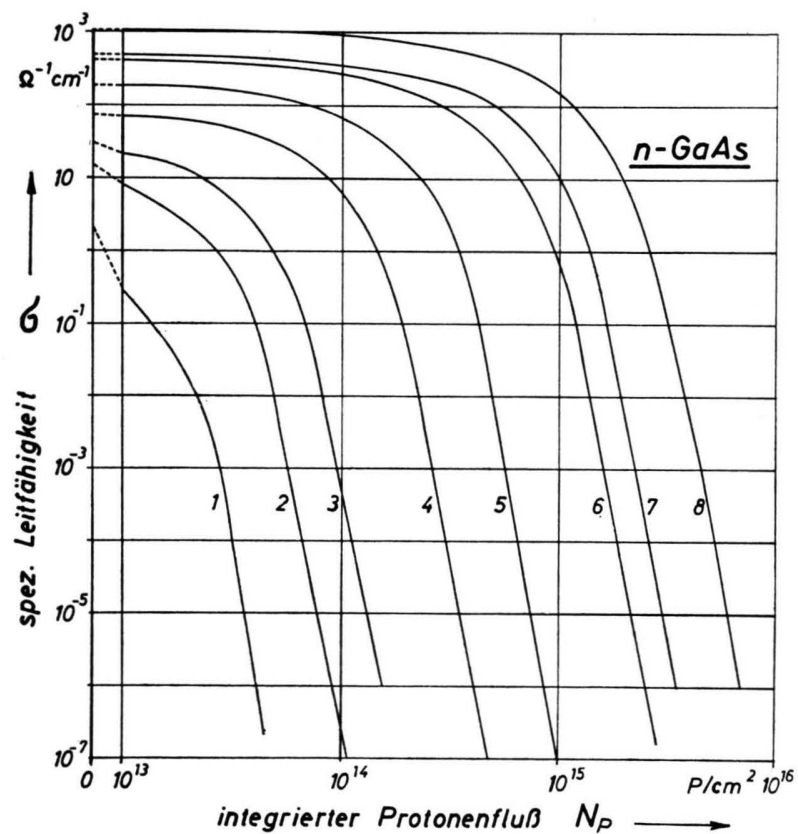

Abb. 4. Spezifische Leitfähigkeit von $n-G a A s$ verschiedener Ausgangsdotierung in Abhängigkeit vom integrierten Protonenfluß bei $3 \mathrm{MeV}$. weitgehend unabhängig von $n_{0}$. Für eine mittlere Probendicke von $45,5 \mu$ ergibt sich damit ein maximal meßbarer Probenwiderstand $R_{\mathrm{n}}^{\max } \approx 2 \cdot 10^{9} \Omega$.

Die durch Bestrahlung verursachte Abnahme der Konzentration und der Beweglichkeit der Elektronen ist unabhängig vom Dotierungselement $(\mathrm{Si}, \mathrm{Te})$, vom Herstellungsverfahren (im Tiegel oder tiegelfrei gezogene Stäbe), sowie vom verwendeten Kontaktmaterial (Sn, In). Es besteht ferner kein Unterschied zwischen geätzten und ungeätzten Proben. Auf den Proben kann also keine durch Ätzen permanent entfernbare leitende Oberflächenschicht vorhanden sein, deren Widerstand mit dem der Proben vergleichbar ist. Dieses Verhalten spricht ebenso wie die offensichtliche Parallelität der Kurven verschiedener Ausgangsdotierung für die Annahme, daß das beobachtete Verhalten von $\mathrm{n}-\mathrm{GaAs}$ bei Protonenbestrahlung tatsächlich den Veränderungen im Kristall (bulk) selbst zuzuschreiben ist, unbeeinflußt von leitenden Oberflächenschichten oder irgendwelchen Zufälligkeiten des verwendeten Materials.

$$
p-G a A s
$$

Wie die Abb. 5 zeigt, verläuft die Abnahme der Trägerkonzentration von $\mathrm{p}-\mathrm{GaAs}$ bei Bestrahlung zunächst ähnlich wie bei n-GaAs. Sobald aber die Löcherkonzentration Werte von $10^{12}-10^{13} \mathrm{~cm}^{-3} \mathrm{er}$ reicht hat, wird - im Unterschied zu n-GaAs der Abfall von $p$ mit zunehmender Bestrahlung schwächer. Der Kurvenverlauf läßt auch für p-GaAs vermuten, daß die Löcherkonzentration durch weitere Bestrahlung noch abnehmen wird; aus den bereits genannten Gründen waren aber keine Messungen mehr möglich. 

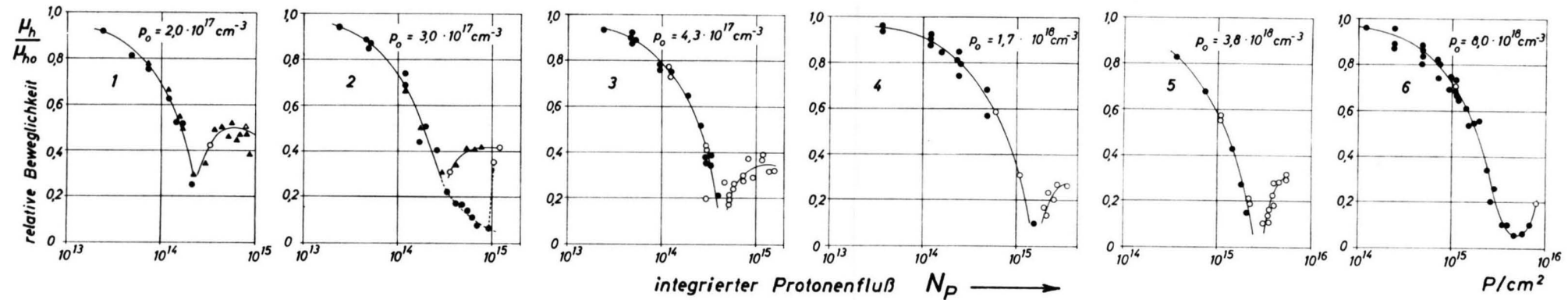

Abb. 6. Beweglichkeit der Löcher in p-GaAs verschiedener Ausgangsdotierung in Abhängigkeit vom integrierten Protonenfluß bei 3 MeV. ätzt; o Sn/Zn-Kontakt, geätzt; $\boldsymbol{\Delta}$ In-Kontakt, nicht geätzt; $\triangle$ In-Kontakt, geätzt.)

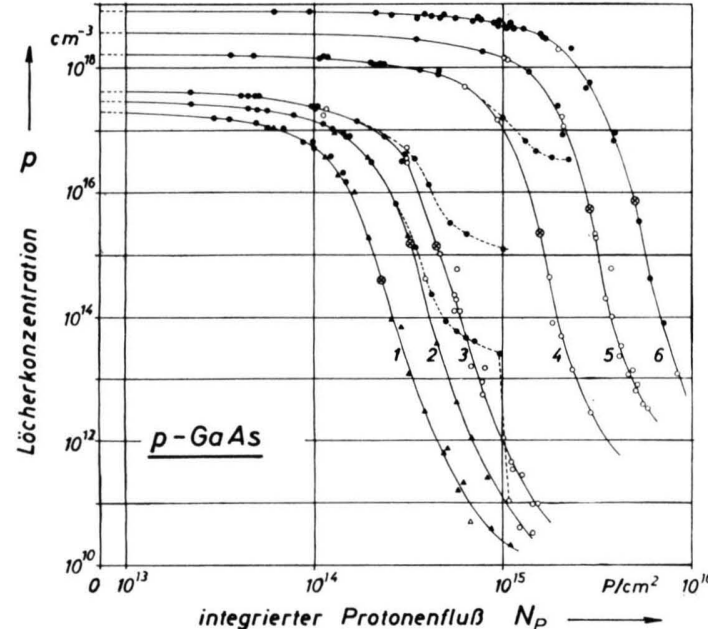

Abb. 5. Löcherkonzentration von p-GaAs verschiedener A Ausgangsdotierung in Abhängigkeit vom integrierten Protonenfluß bei $3 \mathrm{MeV}$. ( $\bullet \mathrm{Sn} / \mathrm{Zn}-\mathrm{Kontakt,}$ nicht geätzt; $\circ \mathrm{Sn} / \mathrm{Zn}-\mathrm{Kontakt,}$ geätzt; $\mathbf{X}$ In-Konta

$$
\triangle \text { In-Kontakt, geätzt). }
$$

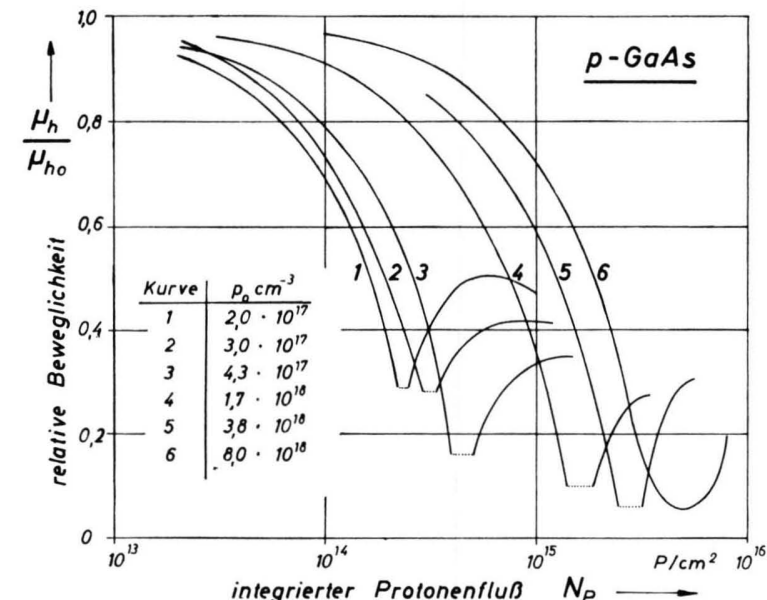

Abb. 7. Beweglichkeit der Löcher in p-GaAs verschiedener Abb. 8. Spezifische Leitfähigkeit von p-GaAs verschiedeusgangsdotierung in Abhängigkeit vom integrierten Proto- ner Ausgangsdotierung in Abhängigkeit vom integrierten nenfluß bei $3 \mathrm{MeV}$ (Zusammenfassung der Abb. 6). ner Ausgangsdotierung in Abhängigkeit
Protonenfluß bei $3 \mathrm{MeV}$.

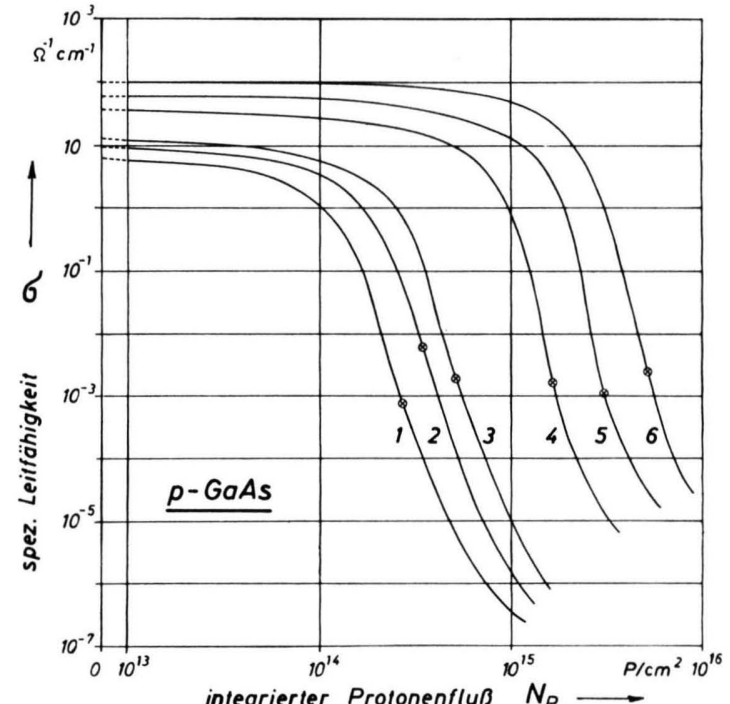


Bei den meisten der mit $\mathrm{Sn} / \mathrm{Zn}$ kontaktierten Proben wurden leitende Oberflächenschichten festgestellt, wodurch ein „Abbiegen“ der Löcherkonzentration auf einen Sättigungswert vorgetäuscht wird. Die in Abb. 5 gestrichelt eingezeichneten Meßkurven von Proben, die mit $\mathrm{Sn} / \mathrm{Zn}$-Kontakten versehen, jedoch nicht geätzt waren, beweisen dies augenfällig. Die Oberflächenschichten stellen einen teilweisen Kurzschluß für die HaLL-Spannung dar. Daraus resultiert eine zu kleine HALL-Konstante bzw. eine zu große Löcherkonzentration und damit auch eine zu kleine Beweglichkeit (Abb. 6), denn durch den Einfluß der Oberflächenschichten sinkt erfahrungsgemäß $R_{\mathrm{H}}$ stärker ab als $\sigma$ steigt. Die 3 Beispiele in Abb. 5 zeigen zugleich, daß die Leitfähigkeit der Oberflächenschichten um Größenordnungen verschieden sein kann. Da auf den mit Sn (bei n-GaAs) oder mit In (bei n- und p-GaAs) kontaktierten Proben dieser Effekt nicht beobachtet wurde, und da Zink eine hohe Oberflächendiffusion besitzt, darf angenommen werden, daß die beobachteten Oberflächenschichten aus Zink bestehen. Nachdem durch Ätzen diese Schichten entfernt wurden, ergaben die Messungen von HALL-Effekt und Leitfähigkeit stets die „normalen“ Werte, wie sie für die bereits geätzten bzw. mit In kontaktierten Proben der betreffenden Ausgangsdotierung beobachtet wurden. In Abb. 5 und 6 verdeutlicht der (gestrichelte) Übergang vom "Sättigungswert“ zum „normalen“ Wert (der Kurve 2) den Einfluß, den das Entfernen der leitenden Zn-Schicht auf das Meßergebnis hat.

Die bei allen Kurven der Abb. 5 beobachtete Tendenz zur Sättigung von $p$ nach starker Bestrahlung, sobald $p$ Werte von $10^{12}-10^{13} \mathrm{~cm}^{-3}$ erreicht hat, könnte ebenfalls durch leitende Schichten, die sich zum Beispiel während der Bestrahlung auf der bereits geätzten Oberfläche ausbilden, vorgetäuscht werden. Deshalb wurde ein Teil der Proben mit $p<10^{12}-10^{13} \mathrm{~cm}^{-3}$ nach der elektrischen Messung nochmals geätzt und anschließend wieder gemessen. Die Ergebnisse vor und nach dem Ätzen stimmten innerhalb der Meßgenauigkeit stets überein. Die Tendenz zur Sättigung von $p$ wird also nicht durch dauernd entfernbare leitende Oberflächenschichten verursacht. Die Möglichkeit, daß sich nach dem Ätzen stets wieder eine Oberflächenschicht mit annähernd gleicher Leitfähigkeit regeneriert, läßt sich zwar grundsätzlich nicht ausschließen, sie erscheint aber wegen des kaum reproduziert durchgeführten
Ätz- und Reinigungsverfahrens wenig wahrscheinlich.

Die Abb. 6 zeigt die durch Bestrahlung verursachte Änderung der Löcherbeweglichkeit in p-GaAs verschiedener Ausgangsdotierung. Die Kurven wurden in Abb. 7 zusammengefaßt. Als gemeinsames Kennzeichen tritt hervor, daß die Löcherbeweglichkeit zunächst abfällt, dann ein Minimum durchläuft, anschließend wieder ansteigt, um schließlich erneut abzufallen. Dabei verschiebt sich mit zunehmender Ausgangsdotierung zugleich mit dem ersten Abfall der Beweglichkeit auch das Minimum nach größerer Protonendosis, gleichzeitig verläuft der Anstieg immer steiler und das erreichte Zwischenmaximum liegt immer tiefer.

Wie die Abb. 8 zeigt, wird der Verlauf der spezifischen Leitfähigkeit $\sigma$ weitgehend durch $p$ bestimmt. Die Abnahme von $\mu_{\mathrm{h}}$ um maximal eine Größenordnung macht sich dabei nur als Korrektur bemerkbar. Der Zwischenanstieg von $\mu_{\mathrm{h}}$ erscheint in den Kurven von $\sigma$ wegen der doppelt logarithmischen Darstellung nur als kaum erkennbare stufenartige Schleife an der durch $\otimes$ gekennzeichneten Stelle. Der Einfluß ist deshalb so gering, weil der Zwischenanstieg von $\mu_{\mathrm{h}}$ mit dem steilen Abfall von $p$ zusammenfällt. Die Abb. 5 zeigt dies deutlich; dort wurde mit $\otimes$ jener integrierte Protonenfluß und die entsprechende Löcherkonzentration bezeichnet, bei welcher die Beweglichkeit das Minimum durchläuft.

Die untere Meßgrenze für $p$ und $\sigma$ dürfte auch bei p-GaAs durch das Auftreten von Oberflächenschichten mit lokal unterschiedlicher Leitfähigkeit verursacht werden. Im Gegensatz zu n-GaAs sind jedoch bei $p-G a A s$ die kleinsten noch meßbaren Werte für $p$ und $\sigma$ stark abhängig von $p_{0}$. Die Werte liegen zwischen $2,5 \cdot 10^{-7} \Omega^{-1} \mathrm{~cm}^{-1}$ für $p_{0}$ $=2 \cdot 10^{17} \mathrm{~cm}^{-3}$ und $3 \cdot 10^{-5} \Omega^{-1} \mathrm{~cm}^{-1}$ für $p_{0}=8,0$ $\cdot 10^{18} \mathrm{~cm}^{-3}$. Daraus ergeben sich maximal meßbare Probenwiderstände $R_{\mathrm{p}}^{\max }$ zwischen $0,9 \cdot 10^{9} \Omega$ und $0,7 \cdot 10^{7} \Omega$. Es läßt sich aber nicht entscheiden, ob die starke Abhängigkeit von $p_{0}$ tatsächlich durch den direkten Einfluß der Ausgangsdotierung auf die leitenden Oberflächenschichten verursacht wird, oder ob der Effekt auf der durch die Ausgangsdotierung bedingten unterschiedlichen Protonendosis beruht, d. h. auf dem Einfluß der Protonenbestrahlung auf die leitenden Oberflächenschichten. Da aber die hochdotierten Proben beim Erreichen der Meßgrenze mit einer nur 7-fach größeren Protonendosis 
bestrahlt wurden als die schwach dotierten Proben, sind die um 2 Größenordnungen unterschiedlichen Werte für $R_{\mathrm{p}}^{\max }$ nicht durch Oberflächenschichten erklärbar, deren Leitfähigkeit proportional zum integrierten Protonenfluß ansteigt.

Die Abnahme von $p$ und die Änderung von $\mu_{\mathrm{h}}$ sind unabhängig vom Dotierungselement (Cd, $\mathrm{Zn}$ ) und vom verwendeten Kontaktmaterial. Der beobachtete Einfluß der Sn/Zn-Kontakte beruht offensichtlich auf einer leitenden Oberflächenschicht aus Zn. Durch Ätzen konnte diese Schicht entfernt werden. Weder durch wiederholtes Ätzen der mit Sn/Zn kontaktierten Proben noch durch erstmaliges Ätzen der mit In kontaktierten Proben lassen sich die Meßergebnisse beeinflussen. Diese Beobachtungen und ebenso die Parallelität im Verhalten der Proben verschiedener Ausgangsdotierung sprechen dafür, daß die Abb.5-8 das tatsächliche Leitfähigkeitsverhalten von $\mathrm{p}-\mathrm{GaAs}$ bei Protonenbestrahlung wiedergeben. Insbesondere ist anzunehmen, daß die beobachtete Tendenz zur Sättigung der Löcherkonzentration nach starker Bestrahlung ebenfalls eine Eigenschaft des Grundmaterials darstellt.

\section{Die Defektdichte in GaAs bei Protonen- bestrahlung}

Die Protonen verlieren in GaAs ihre Energie praktisch ausschließlich durch Wechselwirkung mit der Atomhülle der Gitterbausteine. Die Abhängigkeit der Protonenenergie $E_{\mathrm{P}}$ von der Eindringtiefe in $\mathrm{GaAs}$ läßt sich mit der Formel von Bethe und BLoch für den differentiellen Energieverlust berechnen ${ }^{7,8}$.

Neben der Ionisation erzeugen die Protonen durch elastische Kernstreuung jene Gitterdefekte, die hier primär interessieren. Falls die bei einem solchen Streuprozeß auf das Gitteratom übertragene Energie $E_{\mathrm{T}}$ die Verlagerungsenergie $E_{\mathrm{d}}$ überschreitet, wird dieses Atom von seinem regulären Gitterplatz auf einen Zwischengitterplatz verlagert. Gleichzeitig entsteht eine Gitterleerstelle. Da die Protonen mit einer hinreichend großen Restenergie von $1,1 \mathrm{MeV}$ wieder aus der Rückseite der Proben austreten, läßt sich der totale Wirkungsquerschnitt für die Streuung der Protonen unter den verschiedenen Streuwinkeln

7 W. Whaling, Handb. d. Physik 34, 213 [1958].

8 Die Rechnungen wurden mit der DVA Siemens 2002 durch. geführt. Für die Ausarbeitung des Rechenprogramms sind wir Herrn Dr. FRIE zu Dank verpflichtet. nach der Rutherfordschen Streuformel für ein unabgeschirmtes Coulomb-Potential berechnen. Man erhält:

$$
\sigma=\pi e^{4} Z_{\mathrm{P}}^{2} Z_{\mathrm{T}}^{2} \frac{M_{\mathrm{P}}}{M_{\mathrm{T}}} \frac{1}{E_{\mathrm{P}}}\left(\frac{1}{E_{\mathrm{d}}}-\frac{1}{E_{\mathrm{T}}^{\max }}\right) .
$$

$Z_{\mathrm{P}}, M_{\mathrm{P}}, E_{\mathrm{P}}=$ Kernladungszahl, Masse und Energie des Protons, $Z_{\mathrm{T}}, M_{\mathrm{T}}=$ Kernladungszahl und Masse des Gitteratoms, $E_{\mathrm{T}}^{\max }=$ die im Zentralstoß von einem Proton der Energie $E_{\mathrm{P}}$ auf das Gitteratom übertragbare Energie

$$
\left(=\frac{4 M_{\mathrm{P}} M_{\mathrm{T}}}{\left(M_{\mathrm{P}}+M_{\mathrm{T}}\right)^{2}} E_{\mathrm{P}}\right) .
$$

Da $E_{\mathrm{T}}^{\max } \gg E_{\mathrm{d}}$ allgemein erfüllt ist, gilt für die hier interessierenden Protonenenergien in guter Näherung $\sigma \sim E_{\mathrm{P}}^{-1}$.

Ist $E_{\mathrm{T}}$ hinreichend groß gegen $E_{\mathrm{d}}$, so wird das primär verlagerte Atom durch Stoß mit anderen Gitteratomen diese wiederum verlagern. Je nach der Größe von $E_{\mathrm{T}}$ wird sich dieser Prozeß weiter fortsetzen und zur Bildung einer Verlagerungskaskade führen, die einschließlich des Primärteilchens im Mittel aus $\bar{v}$ Frenkel-Defekten besteht. Aufgrund des Modells von Harrison und SeItz ${ }^{9}$ ergibt sich für $\bar{v}$ folgende Beziehung:

$$
\bar{v}=0,12+0,56 \ln \frac{E_{\mathrm{T}}^{\max }}{E_{\mathrm{d}}} .
$$

Bezeichnet $\eta_{\text {tot }}$ die Zahl aller Gitteratome, die von einem Proton bei konstanter Energie $E_{\mathrm{P}}$ pro Wegelement verlagert werden, so gilt:

$$
\eta_{\text {tot }}=N_{\mathrm{A}} \sigma \overline{\boldsymbol{\nu}} \text {. }
$$

$N_{\mathrm{A}}=$ Zahl der Gitteratome $/ \mathrm{cm}^{3}$.

Die über die gesamte Probendicke $D=45,5 \mu$ gemittelte Defektdichte $\hat{\eta}_{\text {tot }}$ berechnet man nach der Gleichung:

$$
\hat{\eta}_{\text {tot }}=\frac{1}{D} \int_{0}^{D} \eta_{\text {tot }} \mathrm{d} x .
$$

Ebenso ist die über $D$ gemittelte Größe der Verlagerungskaskaden:

$$
\hat{v}=\frac{1}{D} \int_{0}^{D} \bar{v} \mathrm{~d} x .
$$

9 W. A. Harrison u. F. Seitz, Phys. Rev. 98, 1530 [1955]. 
Zur numerischen Berechnung wurde zwischen den eng benachbarten Elementen Ga und As nicht unterschieden. Es wurden „mittlere Atome“ mit $Z_{\mathrm{T}}=32$, $M_{\mathrm{T}}=72,3$ und $N_{\mathrm{A}}=4,42 \cdot 10^{22} / \mathrm{cm}^{3}$ zugrunde gelegt. Für die Verlagerungsenergie $E_{\mathrm{d}}$ liegen zwei Literaturangaben vor. Einmal die von BäUERLEIN ${ }^{10}$ aus der Bildung von Rekombinationszentren bei Elektronenbestrahlung bestimmten Werte $E_{\mathrm{d}}(\mathrm{Ga})=8,8 \mathrm{eV}$ und $E_{\mathrm{d}}(\mathrm{As})=10,1 \mathrm{eV}$; zum anderen der von GRIMSHAw und BANBURY ${ }^{11}$ aus der Abhängigkeit des HaLL-Effekts und des Widerstandes von $\mathrm{n}-\mathrm{GaAs}$ bei Bestrahlung mit Elektronen bei $77^{\circ} \mathrm{K}$ erhaltene Wert $E_{\mathrm{d}}(\mathrm{GaAs})=17-18 \mathrm{eV}$. Die erstere Meßmethode besitzt eine hohe Nachweisempfindlichkeit für Gitterdefekte und liefert deshalb wohl die tatsächliche, d. h. die der Bindungsenergie des Atomgitters entsprechende Verlagerungsenergie. Da aber bei der Verlagerung von Gitteratomen noch andere Energieverluste abzudecken sind, ergibt sich bei Bestrahlung mit höheren Teilchenenergien eine effektive Verlagerungsenergie, die erfahrungsgemäß höher liegt. Dafür dürfte der zweite Wert für $E_{\mathrm{d}}$ repräsentativ sein. Für unsere Überlegungen wurde deshalb $E_{\mathrm{d}}=17,5 \mathrm{eV}$ angenommen. Mit diesen Daten berechnet man für die Grenzwerte der Defektdichten an Vorder- und Rückseite der Proben:

$$
\eta_{\text {tot }}\left(E_{\mathrm{P}}=3,0 \mathrm{MeV}\right)=4,1 \cdot 10^{3} \mathrm{~cm}^{-1}
$$

mit $\bar{v}\left(E_{\mathrm{P}}=3,0 \mathrm{MeV}\right)=5,2$ und

$$
\eta_{\text {tot }}\left(E_{\mathrm{P}}=1,1 \mathrm{MeV}\right)=9,6 \cdot 10^{3} \mathrm{~cm}^{-1}
$$

mit $\quad \overline{\boldsymbol{v}}\left(E_{\mathrm{P}}=1,1 \mathrm{MeV}\right)=4,7$.

Durch Mittelwertbildung über die ganze Probendicke erhält man: $\hat{\eta_{\text {tot }}}=5,8 \cdot 10^{3} \mathrm{~cm}^{-1}$ und $\hat{v}=5,0$ Defekte pro primärem Streuprozeß.

Bezeichnet $q\left(E_{\mathrm{T}}\right)$ die auf $\sigma$ normierte Wahrscheinlichkeit für das Auftreten primär verlagerter Atome mit Energien $\geqq E_{\mathrm{T}}$, d. h. mit Energien zwischen $E_{\mathrm{T}}$ und $E_{\mathrm{T}}^{\max }$, so gilt:

$$
q\left(E_{\mathrm{T}}\right)=\frac{1}{\sigma} \int_{E_{\mathrm{T}}}^{E_{\mathrm{T}}^{\max }}\left(\frac{\mathrm{d} \sigma}{\mathrm{d} E_{\mathrm{T}}}\right) \mathrm{d} E_{\mathrm{T}} .
$$

Nach der RutheRFordschen Streuformel erhält man für den differentiellen Streuquerschnitt

$$
\frac{\mathrm{d} \sigma}{\mathrm{d} E_{\mathrm{T}}}=\pi e^{4} Z_{\mathrm{P}}^{2} Z_{\mathrm{T}}^{2} \frac{M_{\mathrm{P}}}{M_{\mathrm{T}}} \frac{1}{E_{\mathrm{P}}} \frac{1}{E_{\mathrm{T}^{2}}} .
$$

10 R. Bäuerlein, Z. Phys. 176, 498 [1963].

11 J. A. Grimshaw u. P. C. Banbury, Proc. Phys. Soc. London 84, 151 [1964].
Damit berechnet man

$$
q\left(E_{\mathrm{T}}\right)=\frac{E_{\mathrm{d}}\left(E_{\mathrm{T}}^{\max }-E_{\mathrm{T}}\right)}{E_{\mathrm{T}}\left(E_{\mathrm{T}}^{\max }-E_{\mathrm{d}}\right)},
$$

oder für $E_{\mathrm{T}}^{\max } \gg E_{\mathrm{d}}$

$$
q\left(E_{\mathrm{T}}\right) \approx E_{\mathrm{d}}\left(\frac{1}{E_{\mathrm{T}}}-\frac{1}{E_{\mathrm{T}}^{\max }}\right) .
$$

$E_{\mathrm{T}}$ läßt sich durch $v$ ersetzen. Eine übersichtliche Darstellung erhält man, wenn $v$ nicht nach dem Modell von Harrison und SEITz, sondern nach dem einfacheren, im Ergebnis nur wenig abweichenden Modell von KInchin und PEASE ${ }^{12}$ ersetzt wird, gemäß:

$$
v=\left\{\begin{array}{lll}
0 & \text { für } & E_{\mathrm{T}}<E_{\mathrm{d}} \\
1 & \text { für } & E_{\mathrm{d}} \leqq E_{\mathrm{T}} \leqq 2 E_{\mathrm{d}} \\
E_{\mathrm{T}} / 2 E_{\mathrm{d}} & \text { für } & 2 E_{\mathrm{d}} \leqq E_{\mathrm{T}} \leqq E_{\mathrm{i}} \\
E_{\mathrm{i}} / 2 E_{\mathrm{d}} & \text { für } & E_{\mathrm{T}} \leqq E_{\mathrm{i}} .
\end{array}\right.
$$

Mit $E_{\mathrm{i}}$ wird die Mindestenergie der verlagerten Gitteratome bezeichnet, die erforderlich ist, um andere Gitteratome zu ionisieren. Man rechnet also näherungsweise so, als würde der Energieverlust für $E_{\mathrm{T}}>E_{\mathrm{i}}$ ausschließlich durch Ionisation und für $E_{\mathrm{T}}<E_{\mathrm{i}}$ allein durch elastische Kernstreuung erfolgen. Demnach wird durch $E_{\mathrm{i}}$ die maximale Größe der Verlagerungskaskaden bestimmt. Nach einer Faustformel beträgt $E_{\mathrm{i}} \approx M_{\mathrm{T}}(\mathrm{keV})$, in unserem Fall also ca. $72 \mathrm{keV}$. Somit ergibt sich $v_{\max } \approx 2 \cdot 10^{3}$.

Wird mit $q(v)$ die Wahrscheinlichkeit für das Auftreten von Verlagerungskaskaden mit einer Defektzahl $\geqq \nu$ bezeichnet, so erhält man aus Gl. (10) und (12) für $E_{\mathrm{T}}=E_{\mathrm{d}}$ :

$$
q(v)=1 \quad \text { für } \quad v=1
$$

und aus Gl. (11) und (12) für $E_{\mathrm{T}} \geqq 2 E_{\mathrm{d}}$ :

$$
q(v) \approx \frac{1}{2 v}-\frac{E_{\mathrm{d}}}{E_{\mathrm{T}}^{\max }} \text { für } \quad 1 \leqq v \leqq \frac{E_{\mathrm{i}}}{2 E_{\mathrm{d}}} .
$$

Für $2 \mathrm{MeV}$-Protonen in GaAs zum Beispiel ist $E_{\mathrm{T}}^{\max }$ $=108 \mathrm{keV}$ gegenüber $E_{\mathrm{d}}=17,5 \mathrm{eV}$. Nach Gl. (14) erhält man in diesem Fall die folgende Aufstellung über Größe und Häufigkeit der erzeugten Verlagerungskaskaden:

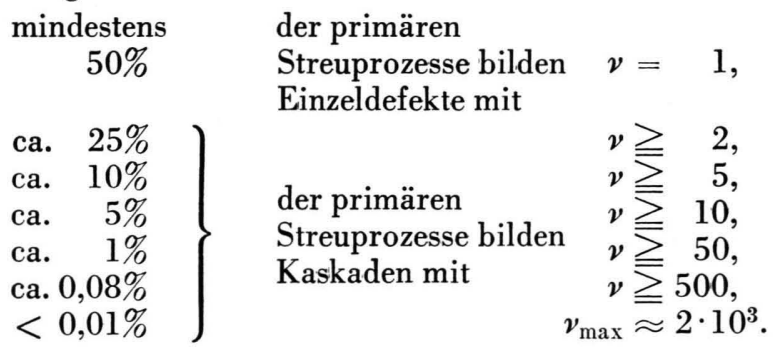


Wegen der großen Reichweite der Coulombschen Wechselwirkung werden bei der Streuung der Protonen an den Gitteratomen kleine Streuwinkel und damit auch kleine Energieübertragungen stark bevorzugt, so daß Einzeldefekte und Verlagerungskaskaden, die nur wenige Defekte umfassen, besonders häufig auftreten. Große Verlagerungskaskaden, sogenannte Cluster, kommen dagegen nur selten vor. Durch die große Zahl der in ihnen enthaltenen Defekte bewirken sie jedoch, daß der Mittelwert $\bar{v}\left(E_{\mathrm{P}}=2 \mathrm{MeV}\right)$ mit 5,0 Defekten pro primärem Streuprozeß relativ hoch ist.

Bei der Defekterzeugung durch energiereiche Protonen sind die Werte für $E_{\mathrm{d}}$ und $E_{\mathrm{T}}^{\max }$ im allgemeinen mit denen des berechneten Beispiels vergleichbar. Die Größe $E_{\mathrm{d}} / E_{\mathrm{T}}^{\max }$, die in Gl. (14) allein die individuellen Daten $\left(E_{\mathrm{d}}, E_{\mathrm{P}}, M_{\mathrm{T}}\right)$ eines gewählten Beispiels enthält, fällt also erst für $\nu>10^{2}$ ins Gewicht. Das heißt aber, daß die obige Aufstellung - von den beiden letzten Zeilen abgesehen - ganz allgemein die angenäherte Verteilung der Gitterdefekte bei Bestrahlung mit Protonen im MeV. Bereich wiedergibt.

Die berechneten Werte für $\eta_{\text {tot }}$ stellen die Summe aller von einem Proton pro Wegelement gebildeten Defekte dar, unabhängig davon, ob die Defekte als Einzeldefekte statistisch im Kristallgitter verstreut oder aber in einem Cluster konzentriert sind. Zur Berechnung von $\eta_{\text {tot }}$ wird also ein Cluster als Summe unabhängiger Einzeldefekte gewertet. Die Vermutung liegt jedoch nahe, daß die Gitterdefekte in beiden Fällen einen unterschiedlichen Einfluß auf die jeweils betrachtete physikalische Meßgröße ausüben. Dies wird auch durch zahlreiche vergleichende Experimente an Halbleitern nach Bestrahlung mit Elektronen und Neutronen bestätigt ${ }^{4,13-15}$. Diese beiden Strahlungsarten stellen gewissermaßen Grenzfälle für die Verteilung strahlungsinduzierter Gitterdefekte dar; denn durch Elektronenbestrahlung werden praktisch ausschließlich isolierte Frenkel-Defekte gebildet, wogegen eine Bestrahlung mit schnellen Neutronen hauptsächlich große Verlagerungskaskaden erzeugt.

\section{Die Träger-Vernichtungsrate}

Einen aufschlußreichen Hinweis auf die energetische Lage der strahlungsinduzierten Energieniveaus 12 G. H. Kinchin u. R. S. Pease, Rept. Prog. Phys. 18, 1 [1955].

13 H. Y. F AN u. K. Lark-Horovitz, The Effects of Radiation on Materials, Reinhold Publ. Corp., New York 1958, S. 159. liefern die Kurven in den Abbildungen 9 und 10, die durch Differentiation der Meßkurven der Abb. 2 und 5 erhalten wurden. Die Größen $\left(-\mathrm{d} n / \mathrm{d} N_{\mathrm{P}}\right)$ für $\mathrm{n}-\mathrm{GaAs}$ und $\left(-\mathrm{d} p / \mathrm{d} N_{\mathrm{P}}\right)$ für $\mathrm{p}-\mathrm{GaAs}$ bezeichnen

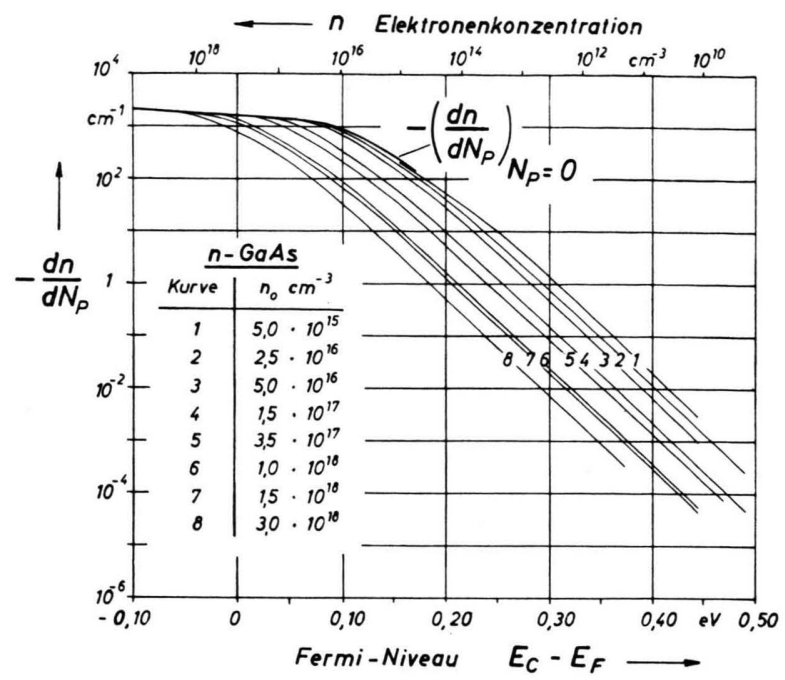

Abb. 9. (Differentielle) Träger-Vernichtungsrate (Abnahme der Elektronenkonzentration pro eingestrahltem Proton $/ \mathrm{cm}^{2}$ ) in $\mathrm{n}-\mathrm{GaAs}$ verschiedener Ausgangsdotierung in Abhängigkeit von der Elektronenkonzentration bzw. von der Lage des FERMI-Niveaus bei $295^{\circ} \mathrm{K}$.

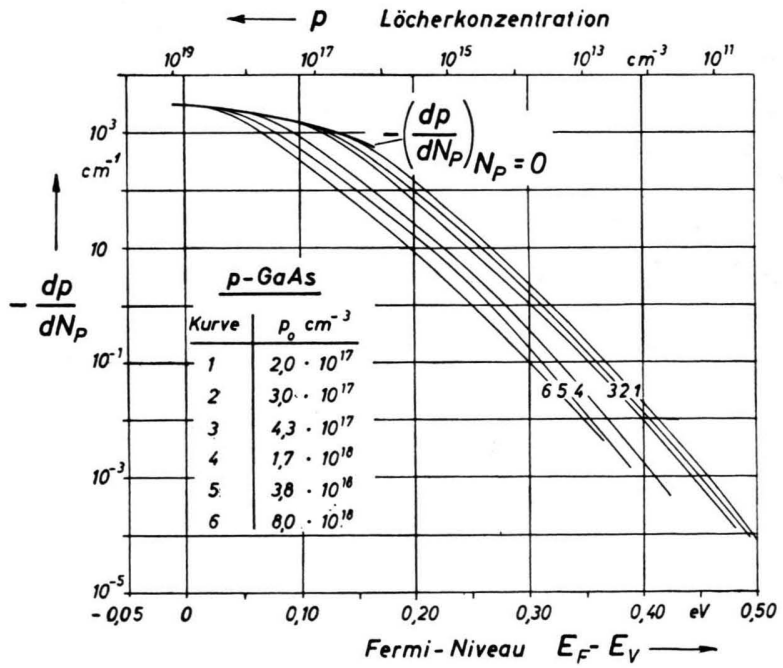

Abb. 10. (Differentielle) Träger-Vernichtungsrate (Abnahme der Löcherkonzentration pro eingestrahltem Proton $/ \mathrm{cm}^{2}$ ) in $\mathrm{p}-\mathrm{GaAs}$ verschiedener Ausgangsdotierung in Abhängigkeit von der Löcherkonzentration bzw. von der Lage des FermINiveaus bei $295^{\circ} \mathrm{K}$.

14 C. A. KLeIN, J. Appl. Phys. 30, 1222 [1959].

15 D. S. Billington u. J. H. Crawford, Radiation Damage in Solids, 1961, Princeton Univ. Press, S. 323-366. 
die Zahl der Ladungsträger $/ \mathrm{cm}^{3}$, die pro eingestrahltem Proton $/ \mathrm{cm}^{2}$ aus dem Leitungs- bzw. Valenzband entfernt werden. Diese Größe wird im folgenden als "Träger-Vernichtungsrate" (carrier removal rate) bezeichnet. Als Abszisse wurde die durch Bestrahlung jeweils schon erreichte Trägerkonzentration sowie die entsprechende Lage des Fermi-Niveaus $E_{\mathrm{F}}$ aufgetragen. Der Abstand des Fermi-Niveaus vom Leitungsband $\left(E_{\mathrm{C}}\right)$ sowie vom Valenzband $\left(E_{\mathrm{V}}\right)$ wurde in bekannter Weise berechnet, gemäß:

$$
\begin{aligned}
& E_{\mathrm{C}}-E_{\mathrm{F}}=k T \ln \frac{N_{\mathrm{C}}}{n} \text { für } \mathrm{n}-\mathrm{GaAs} \\
& \text { mit } n<1,0 \cdot 10^{17} \mathrm{~cm}^{-3}, \\
& E_{\mathrm{F}}-E_{\mathrm{V}}=k T \ln \frac{N_{\mathrm{V}}}{p} \text { für p-GaAs } \\
& \text { mit } p<2,0 \cdot 10^{18} \mathrm{~cm}^{-3} .
\end{aligned}
$$

$T=295^{\circ} \mathrm{K}$, die Probentemperatur bei der elektrischen Messung, $k=$ Boltzmann-Konstante, $N_{\mathrm{C}}, N_{\mathrm{V}}$ $=$ Zustandsdichte im Leitungs- und Valenzband. Für die effektiven Massen $m_{\mathrm{e}}{ }^{*}=0,072 m_{\mathrm{e}}$ und $m_{\mathrm{h}}{ }^{*}=$ $0,5 \mathrm{~m}_{\mathrm{e}}{ }^{16}$ berechnet man $N_{\mathrm{C}}=4,72 \cdot 10^{17} \mathrm{~cm}^{-3}$ bzw. $N_{\mathrm{V}}=8,64 \cdot 10^{18} \mathrm{~cm}^{-3}$.

In dem ebenfalls interessierenden Bereich der Entartung wurde der Zusammenhang zwischen der Lage des Fermi-Niveaus und den Größen $N_{\mathrm{C}} / n$ und $N_{\mathrm{V}} / p$ einer graphischen Darstellung entnommen ${ }^{17}$. Setzt man für die Breite der verbotenen Zone in GaAs $1,35 \mathrm{eV}$, dann erhält man für die Eigenleitungskonzentration $n_{\mathrm{i}}=p_{\mathrm{i}}=6,0 \cdot 10^{6} \mathrm{~cm}^{-3}$ entsprechend $E_{\mathrm{C}}-E_{\mathrm{F}}^{\mathrm{i}_{\mathrm{F}}}=0,638 \mathrm{eV}$ und $E_{\mathrm{F}}^{\mathrm{i}}-E_{\mathrm{V}}=$ $0,712 \mathrm{eV}$.

Wie aus Abb. 9 und 10 ersichtlich, hängt die Träger-Vernichtungsrate entscheidend $a b$ von der Lage des Fermi-Niveaus. Sie ist umso kleiner, je mehr sich $E_{\mathrm{F}}$ zur Mitte des verbotenen Bandes verschiebt. Bei vorgegebener Trägerkonzentration ist die Träger-Vernichtungsrate außerdem umso kleiner, je stärker die betreffende Probe bestrahlt werden mußte, um diese Trägerkonzentration zu erreichen (senkrechter Schnitt in Abb. 9 und 10). Bei vorgegebener Trägerkonzentration besitzt demnach jenes Material die größte Träger-Vernichtungsrate, welches noch nicht vorbestrahlt wurde, für welches also der betrachtete Wert $n$ oder $p$ identisch mit der Ausgangsdotierung $n_{0}$ bzw. $p_{0}$ ist. Die in Abb. 9 und 10 stark ausgezogenen Kurven $(-\mathrm{d} n /$ $\left.\mathrm{d} N_{\mathrm{P}}\right)_{N \mathrm{P}=0}$ und $\left(-\mathrm{d} p / \mathrm{d} N_{\mathrm{P}}\right)_{\mathrm{NP}_{\mathrm{P}}=0}$ kennzeichnen diese

16 C. Hilsum u. A. C. Rose-Innes, Semiconducting III-V-Compounds, Pergamon Press 1961, S. 68 und 118.
Maximalwerte der Träger-Vernichtungsrate bei Bestrahlungsbeginn. Diese beiden Kurven zeigen einen - wenn auch unterschiedlichen - Abfall mit abnehmender Ausgangsdotierung. Bei gleichem Abstand des Fermi-Niveaus von der betreffenden Bandkante ist die Träger-Vernichtungsrate bei Bestrahlungsbeginn für n-GaAs stets um etwa einen Faktor 2 kleiner als bei $\mathrm{p}-\mathrm{GaAs}$.

Für den (willkürlichen) Fall, wenn das FermINiveau gerade mit der betreffenden Bandkante zusammenfällt, ergeben sich folgende Absolutwerte: für $E_{\mathrm{F}}=E_{\mathrm{C}}$ oder $n_{0}=3,9 \cdot 10^{17} \mathrm{~cm}^{-3}$ nach Abb. 9

$$
\left(-\frac{\mathrm{d} n}{\mathrm{~d} N_{\mathrm{P}}}\right)_{\mathrm{N}_{\mathrm{P}}=0}=1,6 \cdot 10^{3} \mathrm{~cm}^{-1}
$$

und für $E_{\mathrm{F}}=E_{\mathrm{V}}$ oder $p_{0}=7,1 \cdot 10^{18} \mathrm{~cm}^{-3}$ nach Abb. 10

$$
\left(-\frac{\mathrm{d} p}{\mathrm{~d} N_{\mathrm{P}}}\right)_{\mathrm{N}_{\mathrm{P}}=0}=3,2 \cdot 10^{3} \mathrm{~cm}^{-1} \text {. }
$$

Zusammen mit der mittleren Verlagerungsdichte $\hat{\eta}_{\text {tot }}$ erhält man für die Zahl $\lambda$ der Ladungsträger, die von jedem nach theoretischen Berechnungen zu erwartenden Frenkel-Defekt unter den genannten Bedingungen aus dem Leitungs- bzw. Valenzband entfernt werden:

$$
\begin{aligned}
\lambda_{\mathrm{n}} & =\frac{1}{\hat{\eta}_{\text {tot }}}\left(-\frac{\mathrm{d} n}{\mathrm{~d} N_{\mathrm{P}}}\right)_{\mathrm{N}_{\mathrm{P}}=0} \text { für } \mathrm{n}-\mathrm{GaAs} \\
& =0,28 \frac{\text { Elektronen }}{\text { Defekt }} \text { für } n_{0}=3,9 \cdot 10^{17} \mathrm{~cm}^{-3}, \\
\lambda_{\mathrm{p}} & =\frac{1}{\hat{\eta}_{\text {tot }}}\left(-\frac{\mathrm{d} p}{\mathrm{~d} N_{\mathrm{P}}}\right)_{\mathrm{N}_{\mathrm{P}}=0} \text { für } \mathrm{p}-\mathrm{GaAs} \\
& =0,55 \frac{\text { Löcher }}{\text { Defekt }} \text { für } p_{0}=7,1 \cdot 10^{18} \mathrm{~cm}^{-3} .
\end{aligned}
$$

Grimshaw und Banbury ${ }^{11}$ haben für n-GaAs von vergleichbarer Ausgangsdotierung bei Bestrahlung mit Elektronen bei Energien von $0,4-1,0 \mathrm{MeV}$ einen Wert $\lambda_{\mathrm{n}}\left(\mathrm{e}^{-}\right) \approx 2$ Leitungselektronen pro Defekt gefunden. Von Aukerman und Graft ${ }^{3}$ wurde bei Bestrahlung von $\mathrm{n}-\mathrm{GaAs}$ mit $1 \mathrm{MeV}$-Elektronen zwar eine um einen Faktor 10 kleinere Träger-Vernichtungsrate bei Bestrahlungsbeginn gemessen. Jedoch kann der Wert nicht zu einem Vergleich herangezogen werden, da bei diesem Experiment die Reichweite der Elektronen und die Probendicke gleich groß waren, so daß - unter Berücksichtigung der Schwellenenergie der Elektronen - in einem Teil des Probenvolumens überhaupt keine Gitterdefekte erzeugt wurden.

17 E. Spenke, Elektronische Halbleiter, Springer-Verlag, Berlin 1955 , S. 304 und 368. 
Bei der Bestrahlung von n-GaAs $\left(n_{0}=10^{16}-\right.$ $10^{17} \mathrm{~cm}^{-3}$ ) mit schnellen Neutronen wurden für die Träger-Vernichtungsrate bei Bestrahlungsbeginn Werte von 3-8 pro $\mathrm{cm}$ und Neutron gemessen ${ }^{1,2,4}$. Aus dem mittleren Streuquerschnitt der Neutronen in $\mathrm{GaAs}$ von 4 barn berechnet man für die Dichte der primären Verlagerungen $\eta_{\text {prim }}=0,17 \mathrm{~cm}^{-1}$. Bei Bestrahlung mit energiereichen Neutronen erzeugt jedes primär verlagerte Gitteratom eine Verlagerungskaskade, die im Mittel aus ca. $10^{3}$ „Einzeldefekten " besteht. Bezogen auf diese Einzeldefekte beträgt $\lambda_{\mathrm{n}}$ (schnelle $\left.\mathrm{n}\right) \approx 0,02$ Leitungselektronen pro Defekt. Wie ein Vergleich zeigt, liegt $\lambda_{\mathrm{n}}$ bei Protonenbestrahlung zwischen $\lambda_{\mathrm{n}}\left(e^{-}\right)$und $\lambda_{\mathrm{n}}$ (schnelle $\mathrm{n})$, jedoch näher bei $\lambda_{\mathrm{n}}\left(e^{-}\right)$. Dieses Ergebnis ist sehr befriedigend, da die räumliche Verteilung der durch Protonenbestrahlung gebildeten Defekte ebenfalls eine Zwischenstellung zwischen den von den beiden anderen Strahlungsarten erzeugten Defektstrukturen einnimmt und gleichzeitig auch näher bei der durch Elektronenbestrahlung gebildeten Defektstruktur liegt (vgl. Abschn. 3). Da $\lambda_{\mathrm{n}}\left(e^{-}\right)$den größten und $\lambda_{\mathrm{n}}$ (schnelle $\mathrm{n}$ ) den kleinsten Wert ergibt, ist zu vermuten, daß die isolierten Einzeldefekte den entscheidenden Beitrag zur Träger-Vernichtungsrate liefern. Die Ursache für den kleinen Wert von $\lambda_{\mathrm{n}}$ (schnelle $\mathrm{n}$ ) dürfte - neben einer möglichen spontanen Rekombination von FrenkelDefekten innerhalb eines Clusters - zumindest teilweise darin bestehen, daß ein Cluster eine stark "bestrahlte" Zone darstellt, in der deshalb die Trägerkonzentration bereits sehr niedrig ist. Aufgrund der vorliegenden Messungen muß in dieser Zone die Träger-Vernichtungsrate und damit auch $\lambda$ entsprechend klein sein.

Im Gegensatz zu der bisher betrachtetenen „differentiellen “ Träger-Vernichtungsrate $\mathrm{d} n / \mathrm{d} N_{\mathrm{P}}$ und $\mathrm{d} p / \mathrm{d} N_{\mathrm{P}}$ möge die auf die Ausgangsdotierung bezogene Größe $\left(n_{0}-n\right) / N_{\mathrm{P}}$ und $\left(p_{0}-p\right) / N_{\mathrm{P}}$ als „integrale“ Träger-Vernichtungsrate bezeichnet werden. Diese Größe stellt gewissermaßen den Mittelwert über die während der gesamten Bestrahlung sich stets verkleinernde differentielle Rate dar. In Abb. 11 wurde die integrale Träger-Vernichtungsrate als Ordinate gegen die durch Bestrahlung jeweils schon erreichte Trägerkonzentration als Abszisse aufgetragen. Die Ordinatenachse trennt bei $\sqrt{n_{\mathrm{i}}{ }^{2}}=6,0 \cdot 10^{6} \mathrm{~cm}^{-3}$ die Bereiche für n- und p-GaAs. Durch diese Darstellung erhält man für n-GaAs eine gute Übereinstimmung der Kurven verschiedener

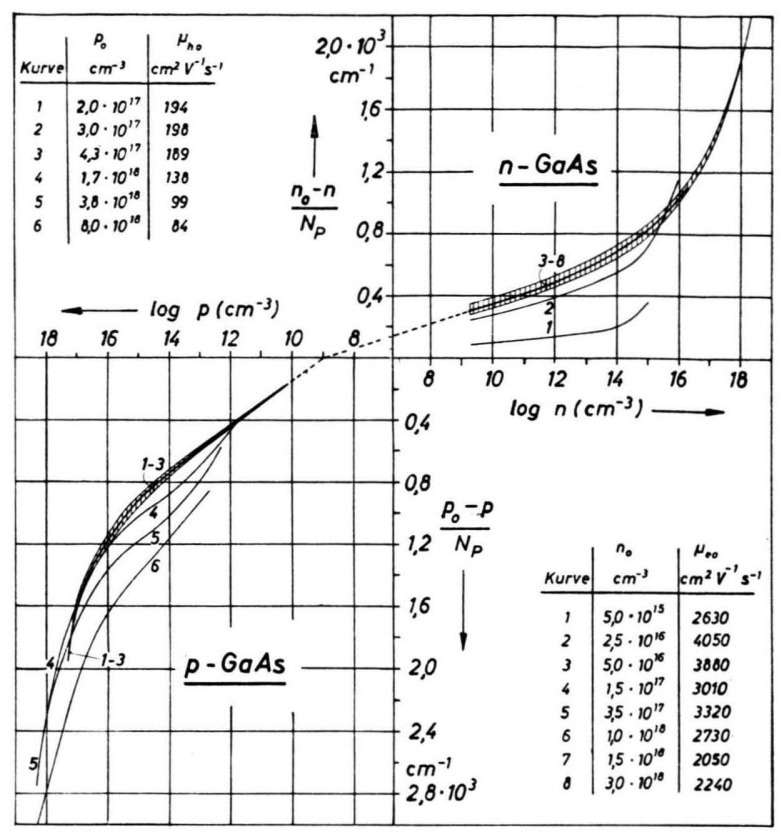

Abb. 11. Auf den integrierten Protonenfluß bezogene (integrale) Träger-Vernichtungsrate in n- und $\mathrm{p}-\mathrm{GaAs}$ verschiedener Ausgangsdotierung in Abhängigkeit von der Ladungsträgerkonzentration bei Bestrahlung mit $3 \mathrm{MeV}$-Protonen.

Ausgangsdotierung. Der schraffierte Bereich umfaßt stets die maximal auftretenden Abweichungen von der durch arithmetische Mittelwertbildung berechneten und ebenfalls eingezeichneten mittleren Kurve. Lediglich die beiden Kurven 1 und 2 mit den kleinsten Ausgangsdotierungen weichen stärker ab. Diese Abweichung beruht bei der Kurve 1 wohl mit Sicherheit und bei Kurve 2 sehr wahrscheinlich darauf, daß es sich in beiden Fällen um stark gegendotiertes Ausgangsmaterial handelt. Die besonders kleine Ausgangsbeweglichkeit der Proben der Kurve 1 legt diese Vermutung nahe.

Bei p-GaAs erhält man eine sehr gute Übereinstimmung der Kurven $1-3$. Dagegen zeigen die Kurven 4-6 mit wachsender Ausgangsdotierung eine zunehmend größere Abweichung von den Kurven $1-3$. Dieser Effekt wird vermutlich nicht allein durch die unterschiedliche Vorbestrahlung, sondern auch durch die unterschiedliche Dotierung bestimmt; denn die Proben der Kurven $1-3$ sind mit Cd und die der Kurven 4-6 mit Zn dotiert.

Die Abb. 11 erlaubt eine Extrapolation auf den bei fortgesetzter Protonenbestrahlung zu erwartenden Grenzwert der Trägerkonzentration. Der Wert $\left(n_{0}-n\right) / N_{\mathrm{P}}=0$ bzw. $\left(p_{0}-p\right) / N_{\mathrm{P}}=0$ kann ja nur für $N_{\mathrm{P}} \Rightarrow \infty$ erreicht werden. Durch lineare Extra- 
polation führen alle Kurven von n-GaAs übereinstimmend auf dieselbe Löcherkonzentration $p^{*} \approx 1 \cdot 10^{9}$ $\mathrm{cm}^{-3}$ als Grenzwert. D. h., daß n-GaAs bei fortgesetzter Protonenbestrahlung zu p-GaAs konvertiert, wobei sich das Fermi-Niveau auf den Grenzwert $E_{\mathrm{F}}^{*}-E_{\mathrm{V}} \approx 0,58 \mathrm{eV}$ einstellt. Dieselbe Grenzkonzentration erhält man auch durch lineare Extrapolation der Kurven $1-3$ von p-GaAs. Dagegen scheinen die Kurven 4-6 größere Grenzwerte von etwa $10^{10} \mathrm{~cm}^{-3}$ zu ergeben. Die überraschend gute Übereinstimmung der extrapolierten Grenzkonzentrationen für n- und p-GaAs ist zwar kein Beweis, aber doch ein Hinweis dafür, daß die Extrapolation in der ausgeführten Weise eine physikalische Berechtigung hat.

Um die zu erwartende Konversion von $\mathrm{n}$ - in p-GaAs möglicherweise auch experimentell zu bestätigen, wurden n-GaAs-Proben $\left(n_{0}=2,5 \cdot 10^{16} \mathrm{~cm}^{-3}\right)$ mit maximal $2,5 \cdot 10^{15}$ Protonen $/ \mathrm{cm}^{2}$ bestrahlt. Die Proben trugen $4 \mathrm{Sn}$ - und $4 \mathrm{Sn} / \mathrm{Zn}$-Kontakte, die jeweils nur auf n-GaAs bzw. nur auf p-GaAs leiten. Sobald durch die Bestrahlung $n<10^{9} \mathrm{~cm}^{-3}$ erreicht war, konnte jedoch über keine der vier Kontakte ein HaLL-Effekt mehr gemessen werden. Ein positives Ergebnis war allerdings auch kaum zu erwarten, da Löcherkonzentrationen unter $10^{10} \mathrm{~cm}^{-3}$ erfahrungsgemäß nicht mehr gemessen werden konnten (vgl. Abschn. 1).

\section{Diskussion}

Das beobachtete Verhalten von GaAs bei Bestrahlung wird durch strahlungsinduzierte Störterme (Energieniveaus) verursacht. Aus den vorliegenden Ergebnissen lassen sich gewisse Aussagen über die energetische Lage dieser Niveaus im verbotenen Band ableiten. Da die Trägerkonzentration von nund p-GaAs bei Bestrahlung stets abnimmt, müssen in GaAs Störterme sowohl mit Donator- als auch mit Akzeptorcharakter erzeugt werden. Die in $\mathrm{GaAs}_{\mathrm{s}}$ sich einstellende Trägerkonzentration läßt sich in diesem allgemeinen Fall nach den Methoden der Reaktionskinetik berechnen ${ }^{17}$. Dabei wird vorausgesetzt, daß in $\mathrm{n}-\mathrm{GaAs}$ alle chemischen Donatoren und in $\mathrm{p}-\mathrm{GaAs}$ alle chemischen Akzeptoren vollständig ionisiert sind.

Es gelten die drei Massenwirkungsgesetze:

$$
\begin{aligned}
D^{+} n & =K_{\mathrm{CD}} \mathrm{D}^{0}, \\
A^{-} p & =K_{\mathrm{AV}} \mathrm{A}^{0}, \\
n p & =N_{\mathrm{C}} N_{\mathrm{V}} e^{-\left(E_{\mathrm{C}}-E_{\mathrm{v}}\right) / k T} .
\end{aligned}
$$

$D^{\mathbf{0}}, A^{\mathbf{0}}=$ Konzentration der mit Elektronen bzw. mit Löchern besetzten (neutralen) Donatoren bzw. Akzeptoren.

$D^{+}, A^{-}=$Konzentration der ionisierten Donatoren bzw. Akzeptoren. Die Konstanten $K$ der Massenwirkungsgesetze ergeben sich aufgrund der BoLTzMANN-Statistik:

$$
\begin{array}{lll}
K_{\mathrm{CD}}=N_{\mathrm{C}} e^{-\left(E_{\mathrm{C}}-E_{\mathrm{D}}\right) / k T} & \text { für } & n \ll N_{\mathrm{C}}, \\
K_{\mathrm{AV}}=N_{\mathrm{V}} e^{-\left(E_{\mathrm{A}}-E_{\mathrm{V}}\right) / k T} & \text { für } & p \ll N_{\mathrm{V}} .
\end{array}
$$

$E_{\mathrm{D}}$ und $E_{\mathrm{A}}$ bezeichnen die Energieniveaus der Donatoren bzw. Akzeptoren. Es gelten ferner zwei Störstellenbilanzen:

$$
\begin{aligned}
& D^{0}+D^{+}=D, \\
& A^{0}+A^{-}=A .
\end{aligned}
$$

$D, A=$ Konzentration der Donatoren bzw. Akzeptoren, die durch Bestrahlung insgesamt erzeugt werden. Die Neutralitätsbedingung lautet

für n-GaAs: $n_{0}-n=A^{-}-D^{+}$, solange $n \gg p$ ist und

für p-GaAs: $p_{0}-p=D^{+}-A^{-}$, solange $p \gg n$ ist .

Durch Kombination dieser Gleichungen erhält man z. B. für n-GaAs

mit

$$
n_{0}-n=\frac{n A}{n+K_{\mathrm{CA}}}-\frac{K_{\mathrm{CD}} D}{n+K_{\mathrm{CD}}}
$$

$$
K_{\mathrm{CA}}=N_{\mathrm{C}} e^{-\left(E_{\mathrm{C}}-E_{\mathrm{A}}\right) / k T} .
$$

Es wird vorausgesetzt, daß die Konzentration der strahlungsinduzierten Donatoren und Akzeptoren proportional zum integrierten Protonenfluß $N_{\mathrm{P}}$ verläuft, also

$$
D=\alpha_{\mathrm{D}} N_{\mathrm{P}}, \quad A=\alpha_{\mathrm{A}} N_{\mathrm{P}} .
$$

$\alpha_{\mathrm{D}}, \alpha_{\mathrm{A}}=$ Konzentration aller Donatoren mit $E_{\mathrm{D}}$ bzw. aller Akzeptoren mit $E_{\mathrm{A}}$, die pro eingestrahltem Proton $/ \mathrm{cm}^{2}$ erzeugt werden. Die Gl. (22) ergibt dann für die integrale Träger-Vernichtungsrate von $\mathrm{n}-\mathrm{GaAs}$

$$
\frac{n_{0}-n}{N_{\mathrm{P}}}=\frac{n \alpha_{\mathrm{A}}}{n+K_{\mathrm{CA}}}-\frac{K_{\mathrm{CD}} \alpha_{\mathrm{D}}}{n+K_{\mathrm{CD}}} .
$$

Gl. (25) läßt sich schließlich noch verallgemeinern auf eine Anzahl von $q$ diskreten Akzeptoren $E_{\mathrm{A}}^{\mathrm{i}}$ und von $r$ diskreten Donatoren $E_{\mathrm{D}}^{\mathrm{j}}$ :

$$
\frac{n_{0}-n}{N_{\mathrm{P}}}=\sum_{i=1}^{q} \frac{n \alpha_{\mathrm{A}}^{\mathrm{i}}}{n+K_{\mathrm{CA}}^{\mathrm{j}}}-\sum_{j=1}^{r} \frac{K_{\mathrm{CD}}^{\mathrm{j}} a_{\mathrm{D}}^{\mathrm{j}}}{n+K_{\mathrm{CD}}^{\mathrm{j}}} .
$$

Für p-GaAs erhält man entsprechend

$$
\frac{p_{0}-p}{N_{\mathrm{P}}}=\sum_{j=1}^{r} \frac{p a_{\mathrm{D}}^{\mathrm{j}}}{p+K_{\mathrm{DV}}^{j}}-\sum_{i=1}^{q} \frac{K_{\mathrm{AV}}^{\mathrm{i}} a_{\mathrm{A}}^{\mathrm{i}}}{p+K_{\mathrm{AV}}^{\mathrm{i}}}
$$


mit

$$
K_{\mathrm{DV}}^{\mathrm{j}}=N_{\mathrm{V}} e^{-\left(E_{\mathrm{D}}^{\mathrm{j}}-E_{\mathrm{V}}\right) / k T} .
$$

Es wurde nun versucht, durch geeignete Wahl der Parameter $E_{\mathrm{A}}^{\mathrm{i}}, E_{\mathrm{D}}^{\mathrm{j}}, \alpha_{\mathrm{A}}^{\mathrm{i}}$ und $\alpha_{\mathrm{D}}^{\mathrm{j}}$ die (aus den Kurven $3-8$ von $n-G a A s$ und $1-3$ von $p-G a A s)$ gemittelten Kurven der Abb. 11 zu approximieren. Es ergab sich, daß zur Annäherung des glatten Verlaufs dieser Meßkurven der Abstand benachbarter Energieniveaus einen Wert von etwa $4 k T(=0,1 \mathrm{eV})$ nicht wesentlich überschreiten darf. Bei größerem Niveauabstand zeigen sich Wendepunkte in dem durch die Gln. (26) und (27) bestimmten Kurvenverlauf, sobald das Fermi-Niveau die Mitte zwischen benachbarten Niveaus durchläuft bzw. mit einem der betreffenden Niveaus zusammenfällt. (Innerhalb eines Energieintervalls von $\pm 2 k T$ werden ja aufgrund der Fermi-Statistik Energieniveaus zu 76\% umgeladen.) Vergrößert man den Abstand benachbarter Niveaus schließlich auf etwa $0,20 \mathrm{eV}$, dann treten in den berechneten Kurven waagrechte Abschnitte immer dann auf, wenn das FermI-Niveau eine von Niveaus freie Energiezone durchläuft. Durch die Protonenbestrahlung müssen in $\mathrm{GaAs}$ die Energieniveaus demnach in einer innerhalb etwa $0,1 \mathrm{eV}$ quasikontinuierlichen Verteilung erzeugt werden. Da sich die Beobachtungen bei n-GaAs über einen Bereich $-0,10 \mathrm{eV} \leqq E_{\mathrm{C}}-E_{\mathrm{F}} \leqq 0,50 \mathrm{eV}$ und bei pGaAs über $-0,01 \mathrm{eV} \leqq E_{\mathrm{F}}-E_{\mathrm{V}} \leqq 0,50 \mathrm{eV}$ erstrekken, muß die Verteilung der strahlungsinduzierten Niveaus außerdem von der jeweils nächstgelegenen Bandkante bis zu einem Abstand von mindestens $0,50 \mathrm{eV}$ von dieser reichen.

Wie die Abb. 11 zeigt, unterscheiden sich die integralen Träger-Vernichtungsraten für n- und p$\mathrm{GaAs}$ bei gleichem Abstand des Fermi-Niveaus von der betreffenden Bandkante um maximal einen Faktor 2. Nach Abb. 11 ist auch zu erwarten, daß der bei fortgesetzter Protonenbestrahlung sich einstellende Grenzwert der Trägerkonzentration für $\mathrm{n}$ - und $\mathrm{p}-\mathrm{GaAs}\left(\mathrm{Cd}\right.$ dotiert) bei $p^{*} \approx 1 \cdot 10^{9} \mathrm{~cm}^{-3}$ liegen wird, entsprechend $E_{\mathrm{F}}^{*} \approx E_{\mathrm{V}}+0,58 \mathrm{eV}$ oder einer Grenzlage des FERMI-Niveaus $0,13 \mathrm{eV}$ unterhalb der Mitte des verbotenen Bandes. Diese beiden Beobachtungen lassen vermuten, daß durch die Bestrahlung die Niveaus im oberen und unteren $\mathrm{Ab}$ schnitt des verbotenen Bandes in vergleichbarer Konzentration erzeugt werden und daß die Verteilungen dieser Niveaus angenähert spiegelbildlich zueinander liegen mit $E_{\mathrm{F}}^{*}$ als Symmetrieebene. Da die differentiellen Träger-Vernichtungsraten umso stär- ker abnehmen, je mehr sich das FermI-Niveau von der betreffenden Bandkante entfernt, darf außerdem gefolgert werden, daß die meisten Energieniveaus unmittelbar unterhalb des Leitungsbandes und unmittelbar oberhalb des Valenzbandes gebildet werden.

Die vorliegenden Messungen erlauben jedoch keine eindeutigen Aussagen darüber, wie die strahlungsinduzierten Donatoren bzw. Akzeptoren sich über das verbotene Band verteilen. Die Zahl der freien Parameter zur Anpassung der nach den Gln. (26) und (27) berechneten Kurven an die Meßergebnisse ist zu groß. Selbst die Frage, ob im oberen Abschnitt des verbotenen Bandes bevorzugt Donatoren und im unteren Bandabschnitt bevorzugt Akzeptoren erzeugt werden, oder ob der umgekehrte Fall vorliegt, kann nicht entschieden werden. Die Rechnungen ergeben für eine solche spiegelbildliche Anordnung der Niveaus genau dasselbe Ergebnis.

Wie die Tab. 2 zeigt, wird das Minimum der Löcherbeweglichkeit bei $\mathrm{p}$-GaAs stets dann durchlaufen, wenn der Abstand des Fermi-Niveaus zum Valenzband zwischen $0,18 \mathrm{eV}$ (bei hoher Ausgangs-

\begin{tabular}{|l|c|c|c|c|}
\hline$N_{0}$ & $\begin{array}{c}p_{0} \\
\mathrm{~cm}^{-3}\end{array}$ & $\begin{array}{c}N_{\mathrm{P}}^{\prime} \\
\text { Prot./cm }\end{array}$ & $\begin{array}{c}p^{\prime} \\
\mathrm{cm}^{-3}\end{array}$ & $\begin{array}{c}E_{\mathrm{F}}^{\prime}-E_{\mathrm{V}} \\
\mathrm{eV}\end{array}$ \\
\hline 1 & $2,0 \cdot 10^{17}$ & $2,3 \cdot 10^{14}$ & $4,0 \cdot 10^{14}$ & 0,254 \\
2 & $3,0 \cdot 10^{17}$ & $3,2 \cdot 10^{14}$ & $1,5 \cdot 10^{15}$ & 0,220 \\
3 & $4,3 \cdot 10^{17}$ & $4,5 \cdot 10^{14}$ & $1,5 \cdot 10^{15}$ & 0,220 \\
4 & $1,7 \cdot 10^{18}$ & $1,58 \cdot 10^{15}$ & $2,2 \cdot 10^{15}$ & 0,211 \\
5 & $3,8 \cdot 10^{18}$ & $2,8 \cdot 10^{15}$ & $5,2 \cdot 10^{15}$ & 0,189 \\
6 & $8,0 \cdot 10^{18}$ & $5,0 \cdot 10^{15}$ & $7,3 \cdot 10^{15}$ & 0,180 \\
\hline
\end{tabular}

Tab. 2. Das Minimum der Löcherbeweglichkeit bei Bestrahlung von $\mathrm{p}-\mathrm{GaAs}$ mit Protonen. $N_{\mathrm{P}^{\prime}}=$ integrierter Protonen$\mathrm{flu} / \mathrm{cm}^{2}$, der erforderlich ist, das Minimum der Löcherbeweglichkeit zu erreichen (Abb. 6 und 7). $p^{\prime}=$ Löcherkonzentration $/ \mathrm{cm}^{3}$ nach Bestrahlung mit $N \mathrm{P}^{\prime}$ Protonen $/ \mathrm{cm}^{2}$ (Abb. 5). $E_{\mathrm{F}^{\prime}}-E_{\mathrm{V}}=$ die der Löcherkonzentration $p^{\prime}$ entsprechende Lage des F ERMI-Niveaus $E_{F^{\prime}}$ oberhalb des Valenzbandes [nach Gl. (15) ].

dotierung) und $0,25 \mathrm{eV}$ (bei kleiner Ausgangsdotierung) liegt. Diese Beobachtung wird als Hinweis dafür gewertet, daß in der unteren Hälfte des verbotenen Bandes Donatoren vorhanden sind. Denn geht man von der Annahme aus, daß der Anstieg der Beweglichkeit durch eine Abnahme der Zahl der geladenen Streuzentren verursacht wird, so folgt, daß geladene Streuzentren sich zu neutralen Zentren umladen müssen, wenn das Fermi-Niveau den Bereich zwischen $0,18 \mathrm{eV}$ und $0,25 \mathrm{eV}$ über dem Valenzband von kleineren Werten kommend überschreitet. Diese Bedingung wird aber nur durch Donatoren erfüllt. Daß die Umladung dieser Dona- 
toren sich nicht in einer stufenartigen Abnahme der Träger-Vernichtungsrate bei $E_{\mathrm{F}}^{\prime}-E_{\mathrm{V}}=0,18-0,25 \mathrm{eV}$ bemerkbar macht, könnte darauf beruhen, daß zahlenmäßig tatsächlich nur wenig solcher Donatoren erzeugt werden, ihr starker Einfluß auf die Löcherbeweglichkeit also einem sehr großen Streuquerschnitt zuzuschreiben ist. Wahrscheinlich handelt es sich dabei um mehrfach ionisierte Donatoren; denn die Träger-Vernichtungsrate ist proportional dem Ionisationsgrad, wogegen der Streuquerschnitt sich quadratisch mit dem Ionisationsgrad ändert. Aufgrund des anomalen Verhaltens der Beweglichkeit verschiedener $\mathrm{A}^{\mathrm{III}} \mathrm{B}^{\mathrm{V}}$-Halbleiter wurde bereits von WeIsBerg ${ }^{18}$ - auch für GaAs - die Existenz von Niveaus mit einem extrem hohen Streuquerschnitt gefordert (mobility killer mit einem Streuquerschnitt von $10^{-10}-10^{-11} \mathrm{~cm}^{2}$ ).

Während bei Protonenbestrahlung nach dem Gesagten eine innerhalb $4 k T$ quasikontinuierliche Verteilung von Niveaus im verbotenen Band zu erwarten ist, wurden von Aukerman u. a. ${ }^{4}$ aus der Temperaturabhängigkeit des HALL-Koeffizienten von $\mathrm{GaAs}$ nach Bestrahlung mit Neutronen drei diskrete Niveaus bei etwa $E_{\mathrm{C}}-0,1, E_{\mathrm{C}}-0,5$ und $E_{\mathrm{V}}+0,6 \mathrm{eV}$ gefunden. Analoge Untersuchungen von Aukerman und GRAFT ${ }^{3}$ an GaAs nach Elektronenbestrahlung ergaben ein Niveau, das etwa $0,12 \mathrm{eV}$ oder mehr unter dem Leitungsband liegt. Andererseits konnten von Vitovskir u. a. ${ }^{5}$ durch Kombination verschiedener Untersuchungsmethoden bei n-GaAs insgesamt

18 L. R. Weisberg,J. Appl. Phys. 33, 1817 [1962].
5 und bei p-GaAs 4 diskrete durch $1 \mathrm{MeV}$-Elektronen erzeugte Niveaus identifiziert werden. Dieses Ergebnis kommt dem hier dargelegten Modell für die durch Protonenbestrahlung gebildeten Niveaus wesentlich näher, insbesondere wenn man berücksichtigt, daß die Mannigfaltigkeit der durch $3 \mathrm{MeV}$-Protonen erzeugten Gitterdefekte größer sein wird als bei $1 \mathrm{MeV}$. Elektronen.

Mit der Vorstellung einer quasikontinuierlichen Verteilung der strahlungsinduzierten Niveaus sind schließlich auch optische Untersuchungen verträglich, die von Paul an $n-G a A s$ nach Protonenbestrahlung durchgeführt wurden ${ }^{\mathbf{1 9}}$. Mit wachsendem integriertem Protonenfluß erfolgt eine zunehmende Absorption im Anschluß an die Bandkante, wodurch der steile Abfall der Absorptionskante des unbestrahlten Materials immer stärker abflacht. Diskrete Absorptionslinien wurden nicht beobachtet. Von Aukerman u. a. ${ }^{4}$ wurde an n-GaAs ein ähnliches Verhalten auch nach Neutronenbestrahlung beobachtet. Die zusätzliche kontinuierliche Absorption auf der langwelligen Seite der Absorptionskante würde in diesem Modell einem Ubergang zwischen den nahe am Valenzband liegenden, mit Elektronen besetzten Niveaus zum Leitungsband entsprechen.

Für die Mithilfe bei den elektrischen Messungen sind wir Fräulein W. Rossner sehr zu Dank verpflichtet. Herrn H. Pfann möchten wir für die Durchführung der Bestrahlungen besonders danken.

19 B. PAUL, private Mitteilung. 